\title{
Mining of prognosis-related genes in cervical squamous cell carcinoma immune microenvironment
}

\author{
Jiong Ma ${ }^{\text {Equal first author, } 1}{ }^{,}$Pu Cheng ${ }^{\text {Equal frrt author, } 1,2}{ }^{2}$ Xuejun Chen ${ }^{1}$, Chunxia Zhou ${ }^{1}$, Wei Zheng ${ }^{\text {Corresp. } 1}$ \\ ${ }^{1}$ Department of Gynecology, Second Affiliated Hospital, Zhejiang University School of Medicine, Hang Zhou, China \\ ${ }^{2}$ Key Laboratory of Tumor Microenvironment and Immune Therapy of Zhejiang Prov-ince, Hang Zhou, China \\ Corresponding Author: Wei Zheng \\ Email address: zhengwei@zju.edu.cn
}

Microsoft Word - manuscript change-tracked.docx Purpose: The aim of this study was to explore the effective immune scoring method and mine the novel and potential immune microenvironment-related diagnostic and prognostic markers for cervical squamous cell carcinoma (CSSC). Materials and Methods: The Cancer Genome Atlas (TCGA) data was downloaded and multiple data analysis approaches were initially used to search for the immune-related scoring system on the basis of Estimation of STromal and Immune cells in MAlignant Tumour tissues using Expression data (ESTIMATE) algorithm. After- wards, the representative genes in the gene modules correlated with immune-related scores based on ESTIMATE algorithm were further screened using Weighted Gene Co-expression Network Analysis (WGCNA) and network topology analysis. Gene functions were mined through enrichment analysis, followed by exploration of the correlation between these genes and immune checkpoint genes. Finally, survival analysis was applied to search for genes with significant association with overall survival and external database was employed for further validation. Results: The immune-related scores based on ESTIMATE algorithm was closely associated with other categories of scores, the HPV infection status, prognosis and the mutation levels of multiple CSCC-related genes (HLA and TP53). 18 new representative immune microenvironment-related genes were finally screened closely associated with patient prognosis and were further validated by the independent dataset GSE44001. Conclusion: Our present study suggested that the immune-related scores based on ESTIMATE algorithm can help to screen out novel immune-related diagnostic indicators, therapeutic targets and prognostic predictors in CSCC. 
1

2

3

4 Jiong $\mathrm{Ma}^{1, *}, \mathrm{Pu}$ Cheng ${ }^{1,2, *}$, Xuejun Chen ${ }^{1}$, Chunxia Zhou ${ }^{1}$, Wei Zheng ${ }^{1, \#}$

$5{ }^{1}$ Department of Gynecology, Second Affiliated Hospital, Zhejiang University School of

6 Medicine, Hangzhou, China

$7 \quad{ }^{2}$ Key Laboratory of Tumor Microenvironment and Immune Therapy of Zhejiang Province

$8{ }^{*}$ These authors contributed equally to this work.

$9 \quad$ \# Correspondence: Wei Zheng

10 E-mail address: zhengwei@zju.edn.cn 
12 Abstract

13 Purpose: The aim of this study was to explore the effective immune scoring method and mine the novel and potential immune microenvironment-related diagnostic and prognostic markers for cervical squamous cell carcinoma (CSSC).

Materials and Methods: The Cancer Genome Atlas (TCGA) data was downloaded and multiple data analysis approaches were initially used to search for the immune-related scoring system on the basis of Estimation of STromal and Immune cells in MAlignant Tumour tissues using Expression data (ESTIMATE) algorithm. Afterwards, the representative genes in the gene modules correlated with immune-related scores based on ESTIMATE algorithm were further screened using Weighted Gene Co-expression Network Analysis (WGCNA) and network topology analysis. Gene functions were mined through enrichment analysis, followed by exploration of the correlation between these genes and immune checkpoint genes. Finally, survival analysis was applied to search for genes with significant association with overall survival and external database was employed for further validation.

Results: The immune-related scores based on ESTIMATE algorithm was closely associated with other categories of scores, the HPV infection status, prognosis and the mutation levels of multiple CSCC-related genes (HLA and TP53). 18 new representative immune microenvironment-related genes were finally screened closely associated with patient prognosis and were further validated by the independent dataset GSE44001.

Conclusion: Our present study suggested that the immune-related scores based on ESTIMATE algorithm can help to screen out novel immune-related diagnostic indicators, therapeutic targets and prognostic predictors in CSCC.

Keywords: cervical carcinoma, TCGA, immune, prognosis

\section{Introduction}

Cervical squamous cell carcinoma (CSCC) is one of the most common malignancies in female 
38

39

40

41

reproductive system, which severely threatens female health and life quality(Marth et al. 2018). CSCC is highly prevalent in developing countries, accounting for $60-90 \%$ of global cases(Chen et al. 2017). Radical hysterectomy is currently considered as the dominant therapy for early-stage cervical cancer(Gil-Moreno \& Magrina 2019; Uppal et al. 2019). With the popularization of cervical cancer screening, the therapeutic efficacy and prognosis of early-stage patients has been greatly improved(Altobelli et al. 2019; Ngo-Metzger \& Adsul 2019). Postoperative relapse and metastasis of CSCC remain the major causes of death in clinical practice(Alvarado-Cabrero et al. 2017; Nanthamongkolkul \& Hanprasertpong 2018). Patients with advanced-stage CSCC generally undergo adjuvant radiotherapy and/or chemotherapy, however, the therapeutic effect seems unsatisfactory(Angeles et al. 2019; Bosque et al. 2018). At present, the International Federation of Gynaecology and Obstetrics (FIGO) staging classification is the major criterion for the prognostic prediction of patients with CSCC(Matsuo et al. 2019). Nevertheless, CSCC patients within similar clinical stage usually show diverse prognostic outcomes. Indeed, we now understand that the natural history of CSCC tumorigenesis is a continuous progress accompanied by a series of gene mutations over time. Based on this, CSCC was considered as a heterogenous collection of diseases, which were regarded as the major cause of anti-cancer treatment resistance and cancer relapse(Bachtiary et al. 2006; Kidd \& Grigsby 2008; Srivastava et al. 2019). As FIGO staging lacks heterogeneity of CSCC, clinical treatment decisions are now made depending on multiple factors including gene expression and mutation status other than traditional clinicopathological features. Thus, there is an urgent need to identify high-risk subgroups for individualized monitoring and optimized postoperative therapy in routine clinical practice.

Given the increasing evidence that various immune cells and inflammatory mediators are closely associated with the development of $\mathrm{CSCC}$, tumor immune microenvironment is drawing accumulating attention nowadays(Piersma 2011). The leukocytes, neutrophils, lymphocytes and macrophages directly contribute to the immune response, which could be easily and conveniently detected(Chen et al. 2019; Heintzelman et al. 2000; Lu et al. 2018; Rangel-Corona et al. 2011). 
65 In addition, several immune checkpoint biomarkers and cytokines have been identified to mediate the crosstalk between cancer cells and stromal microenvironment(De Nola et al. 2019; Otter et al. 2019). In the last decade, various studies have investigated the relationship between the prognosis of patients with primary CSCC and the immunological landscape through highthroughput quantitative measurements of cellular and molecular characteristics(Minion \& Tewari 2018; Punt et al. 2015). These studies revealed the great heterogeneity of the inflammatory/immune response in $\mathrm{CSCC}$, which might determine to a large extent the final outcome of patients(Bachtiary et al. 2006). More recently, several researchers proposed a novel classification based on the immunological status of CSCC according to the ratio of different immune cells (such as monocyte/lymphocyte ratio or Th17/Treg ratio) in the tumor microenvironment, which might play a significant role in the accurate prediction of patient prognosis(Huang et al. 2019; Zhang et al. 2011). Unfortunately, almost none of the previous studies have reached clinical practice because of lacking the exploration from large sample data. On this account, multiple immune scoring methods have been exploited using the expression data of immune-related genes in The Cancer Genome Atlas (TCGA) database which enable us to quantify the immune microenvironment status of a specific patient(Lee et al. 2012; Shen et al. 2018). For instance, the systemic immune-inflammation index (SII) established according to peripheral lymphocyte, neutrophil and platelet counts has been considered as a good indicator reflecting the local immune response and systemic inflammation(Fest et al. 2019). Moreover, SII has been confirmed to have remarkable association with the prognosis of numerous tumors, including non-small cell lung cancer(Guo et al. 2019), esophageal cancer(Ishibashi et al. 2018) and colorectal cancer(Xie et al. 2018). However, there have been only limited studies designed to develop an immune-related prognostic signature for CSCC up to now. Yang et al. established a prognostic immune signature for CSCC based on differential expression analysis and LASSO penalized Cox proportional hazards regression(Yang et al. 2019). However, like many other immune-related models(Cheng et al. 2016; Li et al. 2020; Lu et al. 2020), the variables were screened out based on single gene expression or immune cell proportion. Therefore, despite the 
92 predictive efficiency of these models, their relevance to tumor immune microenvironment is still

93

94 need to be demonstrated. Moreover, among several existing immune-scoring systems, which one is most suitable for CSCC is waiting to be solved.

Earlier published work by Yoshihara group presents a new algorithm that uses the transcriptional profiles of cancer samples to infer the fraction of infiltrating stromal and immune cells, called ESTIMATE(Yoshihara et al. 2013). Importantly, the ESTIMATE method can be broadly applied across almost all human solid cancers. Thus, the ESTIMATE method is a powerful tool for oncologists to elucidate the complex roles of tumor microenvironment and explore potential solutions for tumor heterogeneity.

To this end, our present study was designed to explore the immune scoring method suitable for CSCC. In addition, the gene members in the scoring system were further analyzed by a series of bioinformatic means to mine the novel and potential immune microenvironment-related diagnostic and prognostic markers.

\section{Materials and methods}

\section{Database sources and pre-processing}

The overall flow diagram of present study was summarized in Fig 1. The RNA-seq counts data, SNP data, and clinical follow-up information were downloaded from the TCGA database. The Reads PerKilobase Million (FPKM) data of RNA-Seq were transformed into Transcripts PerKilobase Million (TPM) expression profiles. In consistent with previous studies, 13 metagenes (shown in ImmuneScore.genes.ids.txt, Supplemental File) corresponded to various immunocyte types, reflecting the different immune functions.

\section{Computational methods of multiple immune scores and result determination}

The scores of each sample in the 13 types of metagenes were calculated based on the $\log 2-$ transformed expression of each gene member in the immune metagene (shown in immune.meta.score.txt, Supplemental File)(Safonov et al. 2017). TIMER (https://cistrome.shinyapps.io/timer/) database (immune.immu.score.txt, Supplemental File) was 
119 utilized to calculate the scores of each sample in the immunocyte infiltration (six categories in

120

121

122

123

124

125

126

127

128

129

130

131

132

133

134

135

136

137

138

139

140

141

142

143

144

145

total)(Li et al. 2016). Moreover, the ImmuneScore (that represents the infiltration of immune cells in tumor tissue), StromalScore (that captures the presence of stroma in tumor tissue) and ESTIMATEScore (that infers tumor purity) of each sample (immune.est.score.txt, Supplemental File) were calculated by ESTIMATE function of R software package(Yoshihara et al. 2013). Detailed description of above 3 scores could be found on the website below: https://bioinformatics.mdanderson.org/public-software/estimate/._Finally, $\mathrm{R}$ software package MCPcounter was utilized for the calculation of the abundances of ten immune-related cell (eight categories of immune cells, endothelial cells and fibroblasts) populations in the tumor microenvironment (immune.MCPcounter.score.txt, Supplemental File).

\section{Survival analysis}

Patients were divided into several groups according to each specific parameter (including ImmuneScore, StromalScore, ESTIMATEScore and gene expression level). Afterwards, the association between the gene expression level (or level of ImmuneScore, StromalScore, ESTIMATEScore) and overall survival was analyzed by univariate Cox regression model.

\section{The construction of immune scores-related gene modules through WGCNA}

To begin with, transcripts with over 75\% TPM of $>1$ and median absolute deviation (MAD) of $>$ median were chosen from the expression profile data of all the obtained samples. Hierarchical clustering for cluster analysis of the samples was also adopted. Subsequently, samples with a distance of over 80000 were taken as the outlier samples for screening. Moreover, the distance between any two transcripts was calculated by Pearson correlation coefficient, the establishment of the distance between any two transcripts was performed by the R software package Weighted Gene Co-expression Network Analysis (WGCNA)(Xia et al. 2019), and the soft threshold was set as eight for the screening of the co-expression modules. The co-expression network has been suggested to conform to the scale-free network. In other words, the logarithm of node with the connectivity of $\mathrm{k}(\log (\mathrm{k}))$ should be negatively correlated with the logarithm of the occurrence probability of the specific node $(\log (\mathrm{P}(\mathrm{k}))$, and the correlation coefficient should be $>0.85$. 
146 Proper $\beta$ value was selected in order to ensure the network as a scale-free network. The 147 expression matrix was subsequently transformed into the adjacent matrix, and the latter was 148 further transformed into the topological matrix for gene clustering based on Topological Overlap 149 Matrix (TOM) utilizing the average-linkage hierarchical clustering method in accordance with 150 the mixed dynamic shear tree standard. In addition, the gene number of each gene network

151

152 module was set at least 30 . The dynamic shear method was used to determine the gene module, followed by calculation of the eigengene value of each module in succession. Afterwards, clustering analysis was performed on the modules, in which, modules close to each other were merged into a new module, with re-set appropriate height, deepSplit and minModuleSize values. Finally, the association of the acquired gene modules with ImmuneScore, StromalScore and ESTIMATEScore were separately calculated, in order to explore the gene modules with high correlation for further research.

\section{Establishment of the gene interaction network and functional analysis}

Genes were mapped into the String database(Szklarczyk et al. 2019). The gene-gene interactions were acquired at the score threshold of $>0.4$, followed by visualization using Cytoscape software(Shannon et al. 2003). Meanwhile, Kyoto Encyclopedia of Genes and Genomes (KEGG) and Gene Ontology (GO) enrichment analysis was performed by utilizing the clusterprofile $\mathrm{R}$ package(Xu et al. 2019) to examine the signaling pathways affected by these genes.

\section{Results}

The immune-related scores based on ESTIMATE algorithm is the most suitable immune scoring method for CSCC

To be specific, we retrieved CSCC samples from the TCGA database and analyzed their scores in 23 types of scoring systems, including 13 types of metagenes scores, six types of immunocyte infiltration scores, three types of immune-related scores according to ESTIMATE algorithm (ImmuneScore, StromalScore and ESTIMATEScore) and 10 types of abundances of immunerelated cell. In addition, Spearman's correlation coefficient was used to calculate the correlations 
173 among these scoring systems (shown in Fig.2). As shown in Fig.2A, the average correlation 174 between different types of immune-related scores was greater than 0.4. Among which, 175 ImmuneScore $(\mathrm{R}=0.59)$, Co_inhibition $(\mathrm{R}=0.59)$ and $\mathrm{LCK}(\mathrm{R}=0.62)$ had the highest relevance 176 with other immune scores. These findings showed that there were fine consistency and 177 comparability between different immune scoring systems. The clustering heat maps of various 178 types of scoring systems were shown in Fig.2B, suggesting the great correlation among the 179 scoring systems MHC1, MHC2, Monocytic lineage, Dendritic, Macrophages, ESTIMATEScore, 180 ImmuneScore, Tfh, LCK, Co_stimulation, Co_inhibition, Mete_ImmuneScore, Neutrophil and STAT1. We further investigated the average correlation among immune scores according to four different algorithms. As shown in Fig.2C, the immune-related scores calculated by the ESTIMATE algorithm harbored the highest average correlation with the other three algorithms, which is greater than 0.52 on average. These findings implicated that the immune-related scores based on ESTIMATE algorithm were the most representative immune scoring methods for CSCC.

187 It is widely accepted that HPV infection has a significant association with the occurrence and progression of CSCC(Ding et al. 2019). Therefore, we separately analyzed the ImmuneScore, StromalScore and ESTIMATEScore distribution among CSCC patients with or without HPV infection. As shown in Fig.3A-C, the three immune-related scores in CSCC with HPV infection were significantly higher than those without HPV infection. It should be noted that ImmuneScore was most significantly correlated with the infection status of HPV $(\mathrm{p}<0.05)$.

Subsequently, in order to investigate the association between the above three immune-related scores and prognosis, samples were sorted based on the median of scores of all samples. And then, prognostic difference was analyzed by Kaplan-Meier method (Zou et al. 2007). As a result, the prognosis of samples in different groups was significantly different (shown in Fig.4). And the five-year survival rate of samples with high ImmuneScore and ESTIMATEScore were 198 significantly superior in comparison with those with low scores, suggesting that the three 
200

201

202

203

204

205

206

207

208

209

210

211

212

213

214

215

216

217

218

219

220

221

222

223

224

225

226

novel prognostic markers for CSCC.

A large number of somatic mutations of HLA genes have been reported in CSCC, strongly indicating that loss of function due to HLA mutations is tightly correlated with the immune escape of cancer cells(Xiao et al. 2013). It is of great significance for us to analyze the changes of HLA gene sequence in tumor patients. In addition, the mutation of TP53, a tumor suppressor gene, can induce unlimited proliferation and apoptosis resistance of tumor cells(Laprano et al. 2014; Li et al. 2015). Next, we focused on analyzing the associations of three immune-related scores with mutations of HLA and TP53. To this end, we extracted the mutation data of HLA-A, HLA-B, HLA-C and TP53 from the mutect-processed SNP database and then calculated the three immune-related scores based on ESTIMATE algorithm in HLA-A, HLA-B, HLA-C and TP53 mutation and non-mutation groups. As shown in Fig.5, there was higher level of ImmuneScore in HLA-A and HLA-B mutation groups compared with wild-type groups, while there was also higher level of ESTIMATEScore in HLA-B mutation groups but lower level in TP53 mutation groups comparison with that in wild-type groups.

In summary, we demonstrated that the immune-related scores on the basis of ESTIMATE algorithm were the most proper immune scoring method for CSCC. Additionally, the coexpressed genes with remarkable correlation with these three immune-related scores might be considered as the representative genes in CSCC immune microenvironment, which could be further validated as potential prognostic markers and novel therapeutic targets of CSCC.

\section{Screening of the representative genes in the immune scores-related gene modules}

In this section, clustering analysis was first conducted through hierarchical clustering. As shown in Fig.6A, a total of 296 samples were finally screened out among all the outlier samples, which had a distance of larger than 80000. Subsequently, the weight co-expression network was constructed by WGCNA with $\beta=8$ to guarantee the scale-free network (Fig.6B, C). Afterwards, dynamic shear method(Dong \& Horvath 2007) was utilized to determine the gene modules, and clustering analysis was performed on these modules. Additionally, modules with close distance were further merged into the new module, having height, deepSplit and minModuleSize set to 
227 0.25, 2 and 30, respectively. Finally, a total of 30 modules were acquired (Fig.6D). Of note, the 228 grey module indicated gene sets that could not be clustered into other modules. The transcripts of 229 each module were counted and displayed in Table 1. In total, 6679 transcripts were allocated to 23029 co-expression modules. The correlations of the eigenvectors of these 30 modules with 231 ImmuneScore, StromalScore and ESTIMATEScore were subsequently calculated, respectively. 232 As shown in Fig.6E, the yellow module obviously harbored extremely high association with 233 these three immune-related scores based on ESTIMATE algorithm containing 422 genes.

234 The gene functions in the yellow module were subsequently analyzed. Meanwhile, KEGG and 235 GO enrichment analysis was also conducted using the clusterProfiler of R software package, 236 with flase discovery rate (FDR) set as $<0.05$. The detailed enrichment results were shown as 237 supporting information file (yellow enrich.txt). As a result, the genes in the yellow module were 238 enriched into 50 KEGG pathways, $670 \mathrm{GO}$ biological processes (BP), 85 GO cellular components (CC) and 74 molecular functions (MF). The most significant top 20 KEGG pathways and GO terms were shown in Fig.7. The enriched pathways mainly included Th1 and 241 Th2 cell differentiation, cytokine-cytokine receptor interaction and so on. And the enriched 242 biological processes primarily included T cell activation, leukocyte cell-cell adhesion and so on.

243 The enriched cell components mainly included MHC class II protein complex and T cell receptor complex, and so on. The enriched molecular functions mainly included cytokine receptor activity and MHC class II receptor activity, and the rest. Intriguingly, these enriched pathways and GO term have previously been reported to have close association with CSCC and its immune microenvironment(Roca et al. 2019; Wang et al. 2017; Yasmeen et al. 2010; Zehbe et al. 2005). Finally, to further mine the immune scores-related genes, the weight co-expression relationship between genes in the yellow modules was calculated, with the weight threshold greater than 0.2. Cytoscape software was used for derivation and visualization of the co-expression network of these genes (as shown in Fig.8A). Afterwards, we further analyzed the topological properties of the network, which contained 244 nodes and 4083 edges, indicating that genes with greater association with modules had more close correlation with other genes in the network. As shown 
254 in Fig.8B, the degree distribution of the network was further analyzed, suggesting that the degree

255 of the majority of nodes was extremely small, while the degree of a few nodes was rather large,

256 which was consistent with the characteristics of biological network. The correlation between the

257 gene and the module was further calculated. As shown in Fig.8C, the correlation between most

258 genes and the module was over 0.6, suggesting a high expression similarity between the genes in

259 the module. Moreover, a total of 26 genes (Table 2 and lst.genes.txt as supplemental File) with a

260 correlation over 0.9 and a degree over 50 in the network were selected, with seven members of

261 LCK Metagenes, and one member of Co_inhibition Metagenes. Thus, 18 new representative

262 immune microenvironment-related genes were finally screened.

263 Function analysis of 18 novel representative immune microenvironment-related genes in

264 CSCC patients

265 Firstly, to further analyze the functions of these 18 novel representative immune 266 microenvironment-related genes, the R software package clusterProfiler was utilized for KEGG 267 and GO enrichment analysis, with the significance FDR set at $<0.05$. The detailed results were 268 summarized in lst enrich.txt (supplemental file). In brief, these 18 genes were enriched into 11 269 KEGG pathways, $202 \mathrm{GO}$ biological processes, $8 \mathrm{GO}$ cell components, 19 molecular functions. 270 The most significant 20 KEGG pathways and GO terms were shown in Fig.9, the majority of 271 which were involved in the proliferation, growth and differentiation of $\mathrm{T}$ cells. Intriguingly, 272 LAPTM5, EVI2A and MS4A6A were not enriched in any signaling pathways and GO term, 273 indicating that the functions of these three genes remained completely unclear, which is the focus 274 of our further studies.

275 Secondly, to further investigate the potential roles of the 18 novel representative immune 276 microenvironment-related genes in clinical practice, the R package corrgram was utilized for the 277 calculation of the association between these genes and immune checkpoints (PDCD1、CD274、 278 PDCD1LG2、CTLA4、CD86、CD80、CD276、VTCN1). As shown in Fig.10, apart from 279 CD276 and VTCN1, the other 6 immune checkpoints were significantly related to these 18 genes, 280 with an average correlation coefficient over 0.5 , which indicated that these immune 
281 microenvironment-related genes might be promising targets for immunotherapy.

282 Finally, the prognostic significance of 18 novel representative immune microenvironment-related

283

284

285

286

287

288

289

290

291

292

293

294

295

296

297

298

299

300

301

302

303

304

305

306

307 genes was assessed. According to the median of gene expression, samples were categorized into high and low expression groups. And then the differences of prognosis between these groups were analyzed. As shown in Fig.11, high expression of 13 genes were significantly associated with better overall survival according to the threshold of $p<0.05$, suggesting that these genes might be closely associated with patient prognosis.

Validation of the correlations of 18 immune microenvironment-related genes with ImmuneScore for CSCC patients by using external dataset

External database was used for further validation of the correlations of 18 immune microenvironment-related genes with the immune-related scores according to ESTIMATE algorithm for CSCC patients. Standardized expression matrix was downloaded and extracted from an independent dataset GSE44001(Lee et al. 2013) from Gene Expression Omnibus (GEO). $\mathrm{R}$ packages hgu133plus2.db was utilized to map a probe for gene to extract the expression profiles of these 18 genes, followed by the calculation of the ImmuneScore for each sample using R software package ESTIMATE. Subsequently, the Pearson correlation was calculated between expression of these genes and the level of ImmuneScore for every CSCC sample in this dataset. As shown in Fig.12, apart from CCR5 $(\mathrm{P}=0.867, \mathrm{R}=0.01)$, the other 17 genes were significantly associated with the ImmuneScore, which was consistent with our previous findings.

\section{Discussion}

Great attention has been paid to the association of the immune system with the pathogenesis and progression of tumor in recent years, which has shed light on CSCC therapy, promoting the continuous development of anti-cancer therapy(Dyer et al. 2019; Orbegoso et al. 2018). The external anti-CSCC approaches are frequently applied in previous clinical practice, including surgical resection and chemotherapy. However, the effect of surgical resection is generally restricted due to the invasion into adjacent tissues by cancer cells or distant metastasis. In 
308 addition, the application of chemotherapy is limited due to its toxicity to normal 309 tissues(Menderes et al. 2016). Thus, conventional therapies would exert great burden on the body 310 while providing therapeutic benefits. To this end, it has been widely accepted as a novel 311 direction of anti-cancer therapy by starting from the tumor origin, in other words, the immune 312 system of human body, to control and even kill tumor cells via the modulation of the immune 313 system and enhancement of the anti-tumor immunity in the tumor microenvironment(Ring et al. 314 2017).

315 The tumor microenvironment, mainly composed of immune cells, inflammatory cells, 316 mesenchymal cells, tumor cells, stromal cells, inflammatory mediators and cytokines, provides 317 support for tumor biological behavior including the pathogenesis, progression, invasion and 318 metastasis(Piersma 2011; Qi \& Wu 2019; Tuccitto et al. 2019). Therefore, it is of great 319 significance to discover novel and meaningful immune microenvironment-related genes in 320 CSCC as prognostic predictor and therapeutic targets.

321 In this study, the TCGA database was used to search for the immune microenvironment markers 322 related to the survival time of CSCC patients. And 18 genes were finally detected having 323 remarkable correlation with the prognosis of patients, which was further validated in the GEO 324 database.

325 To be specific, firstly, multiple methods of data analysis were utilized to search for the three 326 immune-related scores on the basis of ESTIMATE algorithm, showing high correlations with 327 diverse other immune-related scores, patients prognosis, HPV infection status and the mutation 328 levels of multiple well-defined CSCC-related genes (HLA and TP53). Secondly, the 329 representative genes in the gene modules associated with immune-related scores according to 330 ESTIMATE algorithm were further searched using WGCNA and network topology analysis. 331 Thirdly, we mined the gene functions through enrichment analysis, followed by the exploration 332 of the association between these genes and immune checkpoint genes. Finally, survival analysis 333 was employed to search for the genes with evident correlation with OS. In addition, external 334 database was employed for further validation of the association of these immune 
335

336

337

338

339

340

341

342

343

344

345

346

347

348

349

350

351

352

353

354

355

356

357

358

359

360

361

microenvironment-related genes with ImmuneScore for CSCC patients. In total, we successfully mined 18 novel potential immune microenvironment-related diagnostic and prognostic indicators or therapeutic targets.

Of note, 11 out of these 18 genes (IL10RA, CD4, HAVCR2, CD2, CCR5, CD3E, BTK, etc) have previously been demonstrated to participate in the pathogenesis, progression, malignant transformation, and pathological process of immune microenvironment of CSCC, which are also significantly associated with patient survival, prognosis and diagnosis(Cao et al. 2013; Che et al. 2016; Hussain et al. 2013; Punt et al. 2015). These above-described observations validate the great reliability and accuracy of the bioinformatic mining results in our present study, in which, we combined TCGA database screening with GEO database for verification. However, the correlations of two genes (LAPTM5 and EVI2A) with CSCC have never been confirmed by any basic or clinical studies, which we are most interested in. LAPTM5, Laptm5, a lysosomal transmembrane protein enhancing the degradation of several targets involved in immune signaling (such as ubiquitin-editing enzyme A20), has been validated to be participate in the modulation of the lethal $\mathrm{T}$ cell alloreactivity mediated by dendritic cells and immunoreactions in multiple inflammatory disease, such as host versus graft disease (GVHD)(Glowacka et al. 2012; Hubbard-Lucey et al. 2014). On the other hand, EVI2A has been confirmed to be involved in lymphocyte proliferation and viability, which is a well-defined immune-specific tumor suppressor in head and neck cancer(Li et al. 2014).

At present, accumulating studies focus on the mining of the association of numerous genes expression with the survival of CSCC patients, however, the majority of previous studies are only performed in animal model, in vitro cell model or small sample samples of tumor patients. Thus, more comprehensive, large-scale population studies are required due to the complexity of CSCC microenvironment. Fortunately, the rapid development of genome-wide sequencing renders the free utilization of high-throughput tumor databases, such as TCGA, making it possible to apply the bioinformatic big data for the large-scale CSCC population. 
362 Conclusion:

363 In the present study, we mainly studied the CSCC immune microenvironment-related gene 364 characteristics. Consequently, these genes are involved in the pathogenesis, progression and 365 malignant transformation of CSCC, affecting OS of CSCC patients. Our present findings can 366 offer more information to decode the complex tumor-tumor interactions in CSCC 367 microenvironment. These findings will help to mine the novel immune-related diagnostic 368 indicators, therapeutic targets and prognostic predictors in CSCC. Besides, the methods of our 369 study have general applicability and provide some references value for the identification of 370 potential diagnostic and prognostic biomarkers for other biologically heterogeneous cancers.

371

372

Author Contribution

373 Wei Zheng: Project development, administration and supervision

374 Jiong Ma: Methodology development, manuscript wrtiing

375 Pu Cheng: Data collection and analysis, manuscript review and editing

376 Xuejun Chen and Chunxia Zhou: data collection, figure organization

378 Funding

379 We gratefully acknowledge the financial support from the Natural Science Fonudation of 380 Zhejiang Province (LY15H040007) and Chinese National Natural Science Foundation 381 (81902629).

382

Compliance with ethical standards

Conflict of interest

385 The authors declare no conflicts of interest in this work.

Ethical approval 
388 This article does not contain any studies with human participants or animals performed by any of 389 the authors.

390 


\section{References}

Altobelli E, Rapacchietta L, Profeta VF, and Fagnano R. 2019. HPV-vaccination and cancer cervical screening in 53 WHO European Countries: An update on prevention programs according to income level. Cancer Med. 10.1002/cam4.2048

Alvarado-Cabrero I, Roma AA, Park KJ, Rutgers JKL, and Silva EG. 2017. Factors Predicting Pelvic Lymph Node Metastasis, Relapse, and Disease Outcome in Pattern C Endocervical Adenocarcinomas. Int J Gynecol Pathol 36:476-485. 10.1097/PGP.0000000000000357

Angeles MA, Baissas P, Leblanc E, Lusque A, Ferron G, Ducassou A, Martinez-Gomez C, Querleu D, and Martinez A. 2019. Magnetic resonance imaging after external beam radiotherapy and concurrent chemotherapy for locally advanced cervical cancer helps to identify patients at risk of recurrence. Int J Gynecol Cancer 29:480-486. 10.1136/ijgc-2018-000168

Bachtiary B, Boutros PC, Pintilie M, Shi W, Bastianutto C, Li JH, Schwock J, Zhang W, Penn LZ, Jurisica I, Fyles A, and Liu FF. 2006. Gene expression profiling in cervical cancer: an exploration of intratumor heterogeneity. Clin Cancer Res 12:5632-5640. 10.1158/1078-0432.CCR-06-0357

Bosque MAS, Cervantes-Bonilla MA, and Palacios-Saucedo GDC. 2018. Clinical and dosimetric factors associated with the development of hematologic toxicity in locally advanced cervical cancer treated with chemotherapy and 3D conformal radiotherapy. Rep Pract Oncol Radiother 23:392-397. 10.1016/j.rpor.2018.07.011

Cao Y, Zhou X, Huang X, Li Q, Gao L, Jiang L, Huang M, and Zhou J. 2013. Tim-3 expression in cervical cancer promotes tumor metastasis. PLoS One 8:e53834. 10.1371/journal.pone.0053834

Che LF, Shao SF, and Wang LX. 2016. Downregulation of CCR5 inhibits the proliferation and invasion of cervical cancer cells and is regulated by microRNA-107. Exp Ther Med 11:503-509. 10.3892/etm.2015.2911

Chen SB, Yang XH, Weng HR, Liu DT, Li H, and Chen YP. 2017. Clinicopathological features and surgical treatment of cervical oesophageal cancer. Sci Rep 7:3272. 10.1038/s41598-017-03593-0

Chen XJ, Wu S, Yan RM, Fan LS, Yu L, Zhang YM, Wei WF, Zhou CF, Wu XG, Zhong M, Yu YH, Liang L, and Wang W. 2019. The role of the hypoxia-Nrp-1 axis in the activation of M2-like tumor-associated macrophages in the tumor microenvironment of cervical cancer. Mol Carcinog 58:388-397. 10.1002/mc.22936

Cheng W, Ren X, Zhang C, Cai J, Liu Y, Han S, and Wu A. 2016. Bioinformatic profiling identifies an immune-related risk signature for glioblastoma. Neurology 86:2226-2234. 10.1212/WNL.0000000000002770

De Nola R, Menga A, Castegna A, Loizzi V, Ranieri G, Cicinelli E, and Cormio G. 2019. The Crowded Crosstalk between Cancer Cells and Stromal Microenvironment in Gynecological Malignancies: Biological Pathways and Therapeutic Implication. Int J Mol Sci 20. 10.3390/ijms20102401

Ding L, Liu C, Zhou Q, Feng M, and Wang J. 2019. Association of estradiol and HPV/HPV16 infection with the occurrence of cervical squamous cell carcinoma. Oncol Lett 17:3548-3554. 10.3892/ol.2019.10005

Dong J, and Horvath S. 2007. Understanding network concepts in modules. BMC Syst Biol 1:24. 10.1186/17520509-1-24

Dyer BA, Zamarin D, Eskandar RN, and Mayadev JM. 2019. Role of Immunotherapy in the Management of Locally Advanced and Recurrent/Metastatic Cervical Cancer. I Natl Compr Canc Netw 17:91-97. 10.6004/jnccn.2018.7108

Fest J, Ruiter R, Mulder M, Groot Koerkamp B, Ikram MA, Stricker BH, and van Eijck CHJ. 2019. The systemic 
431

432

433

434

435

436

437

438

439

440

441

442

443

444

445

446

447

448

449

450

451

452

453

454

455

456

457

458

459

460

461

462

463

464

465

466

467

468

469

470

471 immune-inflammation index is associated with an increased risk of incident cancer-A population-based cohort study. Int J Cancer. 10.1002/ijc.32303

Gil-Moreno A, and Magrina JF. 2019. Minimally Invasive or Abdominal Radical Hysterectomy for Cervical Cancer. N Engl J Med 380:794. 10.1056/NEJMc1816590

Glowacka WK, Alberts P, Ouchida R, Wang JY, and Rotin D. 2012. LAPTM5 protein is a positive regulator of proinflammatory signaling pathways in macrophages. I Biol Chem 287:27691-27702. 10.1074/jbc.M112.355917

Guo W, Cai S, Zhang F, Shao F, Zhang G, Zhou Y, Zhao L, Tan F, Gao S, and He J. 2019. Systemic immuneinflammation index (SII) is useful to predict survival outcomes in patients with surgically resected nonsmall cell lung cancer. Thorac Cancer 10:761-768. 10.1111/1759-7714.12995

Heintzelman DL, Lotan R, and Richards-Kortum RR. 2000. Characterization of the autofluorescence of polymorphonuclear leukocytes, mononuclear leukocytes and cervical epithelial cancer cells for improved spectroscopic discrimination of inflammation from dysplasia. Photochem Photobiol 71:327-332.

Huang H, Liu Q, Zhu L, Zhang Y, Lu X, Wu Y, and Liu L. 2019. Prognostic Value of Preoperative Systemic ImmuneInflammation Index in Patients with Cervical Cancer. Sci Rep 9:3284. 10.1038/s41598-019-39150-0

Hubbard-Lucey VM, Shono Y, Maurer K, West ML, Singer NV, Ziegler CG, Lezcano C, Motta AC, Schmid K, Levi SM, Murphy GF, Liu C, Winkler JD, Amaravadi RK, Rogler G, Dickinson AM, Holler E, van den Brink MR, and Cadwell K. 2014. Autophagy gene Atg16L1 prevents lethal T cell alloreactivity mediated by dendritic cells. Immunity 41:579-591. 10.1016/j.immuni.2014.09.011

Hussain SK, Madeleine MM, Johnson LG, Du Q, Galloway DA, Daling JR, Malkki M, Petersdorf EW, and Schwartz SM. 2013. Nucleotide variation in IL-10 and IL-12 and their receptors and cervical and vulvar cancer risk: a hybrid case-parent triad and case-control study. Int J Cancer 133:201-213. 10.1002/ijc.28000

Ishibashi Y, Tsujimoto H, Hiraki S, Kumano I, Yaguchi Y, Horiguchi H, Nomura S, Ito N, Shinto E, Aosasa S, Yamamoto J, and Ueno H. 2018. Prognostic Value of Preoperative Systemic Immunoinflammatory Measures in Patients with Esophageal Cancer. Ann Surg Oncol 25:3288-3299. 10.1245/s10434-018-6651-y

Kidd EA, and Grigsby PW. 2008. Intratumoral metabolic heterogeneity of cervical cancer. Clin Cancer Res 14:52365241. 10.1158/1078-0432.CCR-07-5252

Laprano TD, Lemos EH, Cunha LM, Junior JE, de SousaTeles RA, and Rabenhorst SH. 2014. Association of TP53 codon 72 and intron 3 16-bp Ins/Del polymorphisms with cervical cancer risk. Tumour Biol 35:7435-7440. 10.1007/s13277-014-1988-8

Lee YY, Choi CH, Sung CO, Do IG, Hub SJ, Kim HJ, Kim TJ, Lee JW, Bae DS, and Kim BG. 2012. Clinical significance of changes in peripheral lymphocyte count after surgery in early cervical cancer. Gynecol Oncol 127:107-113. 10.1016/j.ygyno.2012.05.039

Lee YY, Kim TJ, Kim JY, Choi CH, Do IG, Song SY, Sohn I, Jung SH, Bae DS, Lee JW, and Kim BG. 2013. Genetic profiling to predict recurrence of early cervical cancer. Gynecol Oncol 131:650-654. 10.1016/j.ygyno.2013.10.003

Li B, Severson E, Pignon JC, Zhao H, Li T, Novak J, Jiang P, Shen H, Aster JC, Rodig S, Signoretti S, Liu JS, and Liu XS. 2016. Comprehensive analyses of tumor immunity: implications for cancer immunotherapy. Genome Biol 17:174. 10.1186/s13059-016-1028-7

Li B, Wang X, Chen H, Shang LX, and Wu N. 2015. TP53 codon 72 polymorphism and susceptibility to cervical cancer in the Chinese population: an update meta-analysis. Int J Clin Exp Med 8:9055-9062.

PeerJ reviewing PDF | (2020:02:45770:1:1:NEW 1 Jul 2020) 
472

473

474

475

476

477

478

479

480

481

482

483

484

485

486

487

488

489

490

491

492

493

494

495

496

497

498

499

500

501

502

503

504

505

506

507

508

509

510

511

512

Li F, Guo H, Wang Y, Liu B, and Zhou H. 2020. Profiles of tumor-infiltrating immune cells and prognostic genes associated with the microenvironment of bladder cancer. Int Immunopharmacol 85:106641. 10.1016/j.intimp.2020.106641

Li XW, Rees JS, Xue P, Zhang H, Hamaia SW, Sanderson B, Funk PE, Farndale RW, Lilley KS, Perrett S, and Jackson AP. 2014. New insights into the DT40 B cell receptor cluster using a proteomic proximity labeling assay. J Biol Chem 289:14434-14447. 10.1074/jbc.M113.529578

Lu G, Chen L, Wu S, Feng Y, and Lin T. 2020. Comprehensive Analysis of Tumor-Infiltrating Immune Cells and Relevant Therapeutic Strategy in Esophageal Cancer. Dis Markers 2020:8974793. 10.1155/2020/8974793

Lu XY, Chen M, Chen DH, Li Y, Liu PT, and Liu Y. 2018. Remifentanil on T lymphocytes, cognitive function and inflammatory cytokines of patients undergoing radical surgery for cervical cancer. Eur Rev Med Pharmacol Sci 22:2854-2859. 10.26355/eurrev_201805_14987

Marth C, Landoni F, Mahner S, McCormack M, Gonzalez-Martin A, Colombo N, and Committee EG. 2018. Cervical cancer: ESMO Clinical Practice Guidelines for diagnosis, treatment and follow-up. Ann Oncol 29:iv262. 10.1093/annonc/mdy160

Matsuo K, Machida H, Mandelbaum RS, Konishi I, and Mikami M. 2019. Validation of the 2018 FIGO cervical cancer staging system. Gynecol Oncol 152:87-93. 10.1016/j.ygyno.2018.10.026

Menderes G, Black J, Schwab CL, and Santin AD. 2016. Immunotherapy and targeted therapy for cervical cancer: an update. Expert Rev Anticancer Ther 16:83-98. 10.1586/14737140.2016.1121108

Minion LE, and Tewari KS. 2018. Cervical cancer - State of the science: From angiogenesis blockade to checkpoint inhibition. Gynecol Oncol 148:609-621. 10.1016/j.ygyno.2018.01.009

Nanthamongkolkul K, and Hanprasertpong J. 2018. Predictive Factors of Pelvic Lymph Node Metastasis in EarlyStage Cervical Cancer. Oncol Res Treat 41:194-198. 10.1159/000485840

Ngo-Metzger Q, and Adsul P. 2019. Screening for Cervical Cancer. Am Fam Physician 99:253-254.

Orbegoso C, Murali K, and Banerjee S. 2018. The current status of immunotherapy for cervical cancer. Rep Pract Oncol Radiother 23:580-588. 10.1016/j.rpor.2018.05.001

Otter SJ, Chatterjee J, Stewart AJ, and Michael A. 2019. The Role of Biomarkers for the Prediction of Response to Checkpoint Immunotherapy and the Rationale for the Use of Checkpoint Immunotherapy in Cervical Cancer. Clin Oncol (R Coll Radiol) 31:834-843. 10.1016/j.clon.2019.07.003

Piersma SJ. 2011. Immunosuppressive tumor microenvironment in cervical cancer patients. Cancer Microenviron 4:361-375. 10.1007/s12307-011-0066-7

Punt S, Houwing-Duistermaat JJ, Schulkens IA, Thijssen VL, Osse EM, de Kroon CD, Griffioen AW, Fleuren GJ, Gorter A, and Jordanova ES. 2015. Correlations between immune response and vascularization qRT-PCR gene expression clusters in squamous cervical cancer. Mol Cancer 14:71. 10.1186/s12943-015-0350-0

Qi D, and Wu E. 2019. Cancer prognosis: Considering tumor and its microenvironment as a whole. EBioMedicine. 10.1016/j.ebiom.2019.04.031

Rangel-Corona R, Corona-Ortega T, del Rio-Ortiz I, Nieves-Ramirez ME, Moran-Banuelos H, Gonzalez-Tenorio O, Caceres-Cortes JR, and Weiss-Steider B. 2011. Cationic liposomes bearing IL-2 on their external surface induced mice leukocytes to kill human cervical cancer cells in vitro, and significantly reduced tumor burden in immunodepressed mice. J Drug Target 19:79-85. 10.3109/10611861003733920

Ring KL, Yemelyanova AV, Soliman PT, Frumovitz MM, and Jazaeri AA. 2017. Potential immunotherapy targets in recurrent cervical cancer. Gynecol Oncol 145:462-468. 10.1016/j.ygyno.2017.02.027

Peer) reviewing PDF | (2020:02:45770:1:1:NEW 1 Jul 2020) 
513

514

515

516

517

518

519

520

521

522

523

524

525

526

527

528

529

530

531

532

533

534

535

536

537

538

539

540

541

542

543

544

545

546

547

548

549

550

551

552

553

Roca AM, Chobrutskiy BI, Callahan BM, and Blanck G. 2019. T-cell receptor V and J usage paired with specific HLA alleles associates with distinct cervical cancer survival rates. Hum Immunol 80:237-242. 10.1016/j.humimm.2019.01.005

Safonov A, Jiang T, Bianchini G, Gyorffy B, Karn T, Hatzis C, and Pusztai L. 2017. Immune Gene Expression Is Associated with Genomic Aberrations in Breast Cancer. Cancer Res 77:3317-3324. 10.1158/00085472.CAN-16-3478

Shannon P, Markiel A, Ozier O, Baliga NS, Wang JT, Ramage D, Amin N, Schwikowski B, and Ideker T. 2003. Cytoscape: a software environment for integrated models of biomolecular interaction networks. Genome Res 13:2498-2504. 10.1101/gr.1239303

Shen L, Yu H, Liu M, Wei D, Liu W, Li C, and Chang Q. 2018. A ten-long non-coding RNA signature for predicting prognosis of patients with cervical cancer. Onco Targets Ther 11:6317-6326. 10.2147/OTT.S175057

Srivastava S, Koay EJ, Borowsky AD, De Marzo AM, Ghosh S, Wagner PD, and Kramer BS. 2019. Cancer overdiagnosis: a biological challenge and clinical dilemma. Nat Rev Cancer 19:349-358. 10.1038/s41568019-0142-8

Szklarczyk D, Gable AL, Lyon D, Junge A, Wyder S, Huerta-Cepas J, Simonovic M, Doncheva NT, Morris JH, Bork P, Jensen L, and Mering CV. 2019. STRING v11: protein-protein association networks with increased coverage, supporting functional discovery in genome-wide experimental datasets. Nucleic Acids Res 47:D607-D613. 10.1093/nar/gky1131

Tuccitto A, Shahaj E, Vergani E, Ferro S, Huber V, Rodolfo M, Castelli C, Rivoltini L, and Vallacchi V. 2019. Immunosuppressive circuits in tumor microenvironment and their influence on cancer treatment efficacy. Virchows Arch 474:407-420. 10.1007/s00428-018-2477-z

Uppal S, Rebecca Liu J, Kevin Reynolds R, Rice LW, and Spencer RJ. 2019. Trends and comparative effectiveness of inpatient radical hysterectomy for cervical cancer in the United States (2012-2015). Gynecol Oncol 152:133-138. 10.1016/j.ygyno.2018.09.027

Wang X, Jiang Y, Yuan M, Chen C, Wang K, Zhang Q, Zuo Y, and Ren S. 2017. Overexpression of dendritic cellspecific intercellular adhesion molecule-3-grabbing nonintegrin-related protein in cervical cancer and correlation with squamous cell carcinoma antigen. Oncol Lett 14:2813-2821. 10.3892/ol.2017.6508

Xia WX, Yu Q, Li GH, Liu YW, Xiao FH, Yang LQ, Rahman ZU, Wang HT, and Kong QP. 2019. Identification of four hub genes associated with adrenocortical carcinoma progression by WGCNA. PeerJ 7:e6555. 10.7717/peerj.6555

Xiao X, Liu L, Li WJ, Liu J, and Chen DJ. 2013. HLA-A, HLA-B, HLA-DRB1 polymorphisms and risk of cervical squamous epithelial cell carcinoma: a population study in China. Asian Pac J Cancer Prev 14:4427-4433.

Xie QK, Chen P, Hu WM, Sun P, He WZ, Jiang C, Kong PF, Liu SS, Chen HT, Yang YZ, Wang D, Yang L, and Xia LP. 2018. The systemic immune-inflammation index is an independent predictor of survival for metastatic colorectal cancer and its association with the lymphocytic response to the tumor. J Transl Med 16:273. 10.1186/s12967-018-1638-9

Xu Z, Wang C, Xiang X, Li J, and Huang J. 2019. Characterization of mRNA Expression and Endogenous RNA Profiles in Bladder Cancer Based on The Cancer Genome Atlas (TCGA) Database. Med Sci Monit 25:3041-3060. 10.12659/MSM.915487

Yang S, Wu Y, Deng Y, Zhou L, Yang P, Zheng Y, Zhang D, Zhai Z, Li N, Hao Q, Song D, Kang H, and Dai Z. 2019. Identification of a prognostic immune signature for cervical cancer to predict survival and response to

Peer] reviewing PDF | (2020:02:45770:1:1:NEW 1 Jul 2020) 
554 immune checkpoint inhibitors. Oncoimmunology 8:e1659094. 10.1080/2162402X.2019.1659094

555

556

557

558

559

560

561

562

563

564

565

566

567

568

569
Yasmeen A, Alachkar A, Dekhil H, Gambacorti-Passerini C, and Al Moustafa AE. 2010. Locking Src/Abl Tyrosine Kinase Activities Regulate Cell Differentiation and Invasion of Human Cervical Cancer Cells Expressing E6/E7 Oncoproteins of High-Risk HPV. J Oncol 2010. 10.1155/2010/530130

Yoshihara K, Shahmoradgoli M, Martinez E, Vegesna R, Kim H, Torres-Garcia W, Trevino V, Shen H, Laird PW, Levine DA, Carter SL, Getz G, Stemke-Hale K, Mills GB, and Verhaak RG. 2013. Inferring tumour purity and stromal and immune cell admixture from expression data. Nat Commun 4:2612. 10.1038/ncomms3612

Zehbe I, Hohn H, Pilch H, Neukirch C, Freitag K, and Maeurer MJ. 2005. Differential MHC class II component expression in HPV-positive cervical cancer cells: implication for immune surveillance. Int J Cancer 117:807815. 10.1002/ijc.21226

Zhang Y, Ma D, Zhang Y, Tian Y, Wang X, Qiao Y, and Cui B. 2011. The imbalance of Th17/Treg in patients with uterine cervical cancer. Clin Chim Acta 412:894-900. 10.1016/j.cca.2011.01.015

Zou KH, O'Malley AJ, and Mauri L. 2007. Receiver-operating characteristic analysis for evaluating diagnostic tests and predictive models. Circulation 115:654-657. 10.1161/CIRCULATIONAHA.105.594929 
570 Figure legends

571 Fig.1 Flow diagram of methods for mining of prognostic immune-related genes in CSCC

572 Fig.2 The correlations of immune-related scoring system based on ESTIMATE algorithm 573 with other categories of scores among CSCC samples. (A) The correlations of various 574 immune scoring systems among CSCC samples. Spearman correlation coefficients are shown 575 color-coded to illustrate positive (red) or negative (green) associations. (B) The clustering heat 576 maps of various types of scoring systems. (C) The relationships among immune scores according 577 to four different algorithms. Mete: metagene immune score; Est: ESTIMATE immune score; 578 Timer: Timer immune score; Mcp: MCPcounter immune score.

Fig.3 StromalScore (A), ImmuneScore (B) and ESTIMATEScore (C) distribution among CSCC patients with or without HPV infection.

581 Fig.4 The relationships between levels of StromalScore (A), ImmuneScore (B) or ESTIMATEScore (C) and prognosis for CSCC patients. H: High immune score; L: Low immune score.

Fig.5 The correlations of immune-related scores based on ESTIMATE algorithm with gene mutations. The StromalScore (A, D, G, J), ImmuneScore (B, E, H, K) and ESTIMATEScore (C, F, I, L) were calculated respectively in HLA-A (A, B, C), HLA-B (D, E, F), HLA-C (G, H, I) and TP53 (J, K, L) mutation and non-mutation groups. Green represents the mutant group and red represents the wild type. Mut: Mutant; WT: Wild type.

Fig.6 Immune scores-related gene modules mined through WGCNA. (A) Sample clustering analysis. (B, C) Analysis of network topology for various soft-thresholding powers. (D) Gene dendrogram and module colors. (E) Correlation between each module and three immune-related 592 scores.

593 Fig.7 The KEGG pathway and GO enrichment analysis of the genes in yellow module. (A) 594 Top20 KEGG pathways enriched by the genes in yellow module. (B) Top20 GO BP terms enriched by the genes in yellow module. (C) Top20 GO CC terms enriched by the genes in 596 yellow module. (D) Top20 GO MF terms enriched by the genes in yellow module. GO: Gene 
597 Ontology; BP: biological peocess; CC: cellular component; MF: molecular function

598 Fig.8 Construction of co-expression network of yellow module-related genes. (A) Co599 expression network of weights between genes in yellow module. (B) The degree distribution of 600 nodes in yellow module. (C) The correlation of genes and module in the network.

601

602

603

604

605

606

607
Fig.9 The KEGG pathway and GO enrichment analysis of 18 novel representative immune microenvironment-related genes for CSCC patients. (A) Top20 KEGG pathways enriched by 18 novel representative immune microenvironment-related genes. (B) Top20 GO BP terms enriched by 18 novel representative immune microenvironment-related genes. (C) Top20 GO CC terms enriched by 18 novel representative immune microenvironment-related genes. (D) Top20 GO MF terms enriched by 18 novel representative immune microenvironment-related genes. GO: Gene Ontology; BP: biological process; CC: cellular component; MF: molecular function Fig.10 The association between 18 novel representative immune microenvironment-related genes for CSCC patients and immune checkpoints. Apart from CD276 and VTCN1, the other 6 immune checkpoints were significantly related to 18 immune microenvironment-related genes. Fig.11 The relationship between 18 novel representative immune microenvironmentrelated genes and prognosis $(\mathrm{A}-\mathrm{P})$. Apart from DOCK2 ( $\mathrm{P}=0.12765), \mathrm{SIRPG}(\mathrm{P}=0.0658), \mathrm{CD} 4$ $(\mathrm{P}=0.29192)$, NCKAP1L $(\mathrm{P}=0.12765)$ and CCR5 $(\mathrm{P}=0.06736)$, high expression of other 13 genes were significantly associated with better overall survival.

Fig.12 The correlations of 18 immune microenvironment-related genes with ImmuneScore for CSCC patients in independent dataset (A-P). Apart from CCR5 $(\mathrm{P}=0.867, \mathrm{R}=0.01)$, the other 17 genes were significantly associated with the ImmuneScore.

Peer) reviewing PDF | (2020:02:45770:1:1:NEW 1 Jul 2020) 
Figure 1

Flow diagram of methods for mining of prognostic immune-related genes in CSCC

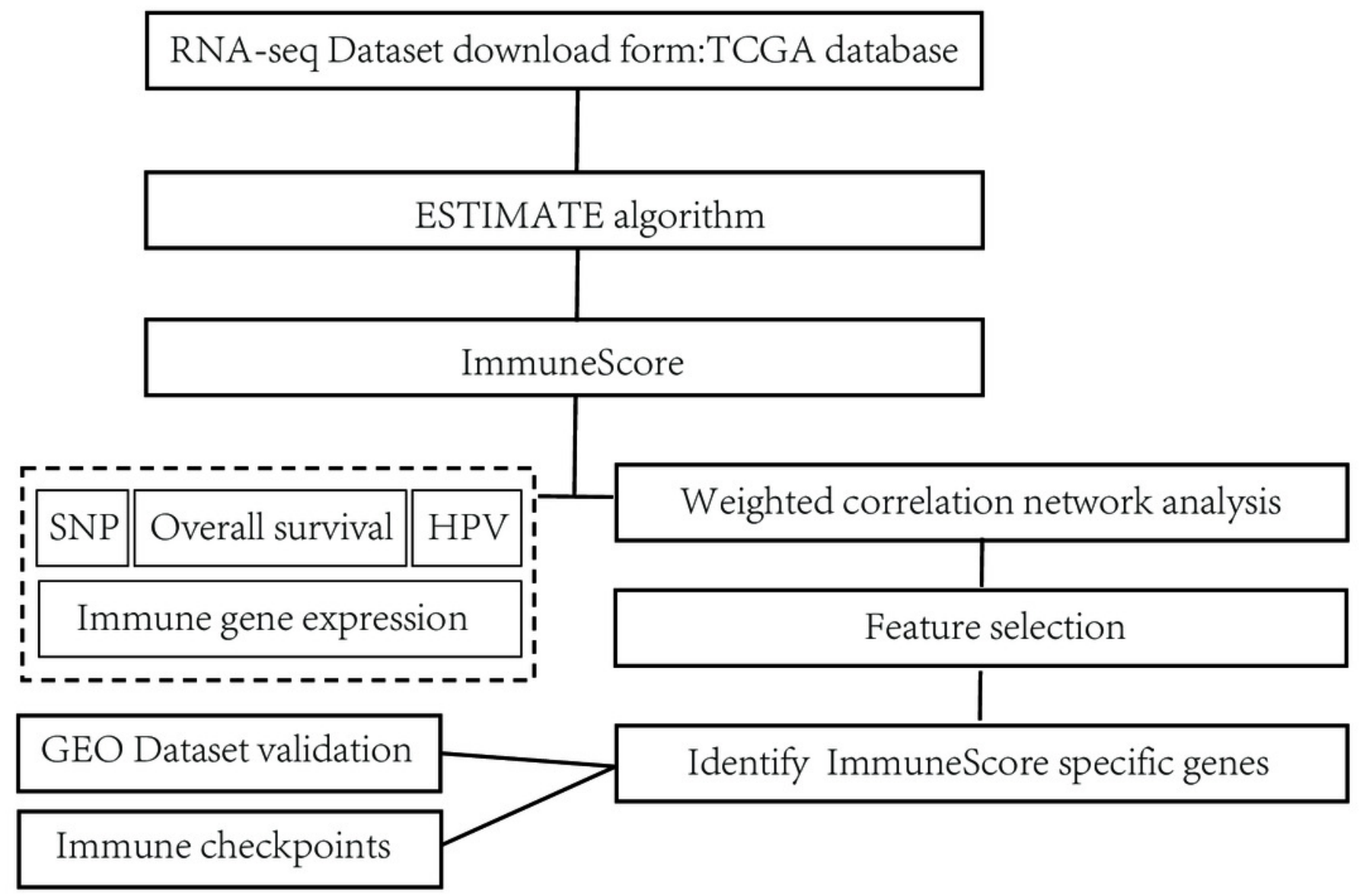




\section{Figure 2}

The correlations of immune-related scoring system based on ESTIMATE algorithm with other categories of scores among CSCC samples.

(A) The correlations of various immune scoring systems among CSCC samples. Spearman correlation coefficients are shown color-coded to illustrate positive (red) or negative (green) associations. (B) The clustering heat maps of various types of scoring systems. (C) The relationships among immune scores according to four different algorithms. Mete: metagene immune score; Est: ESTIMATE immune score; Timer: Timer immune score; Mcp: MCPcounter immune score.
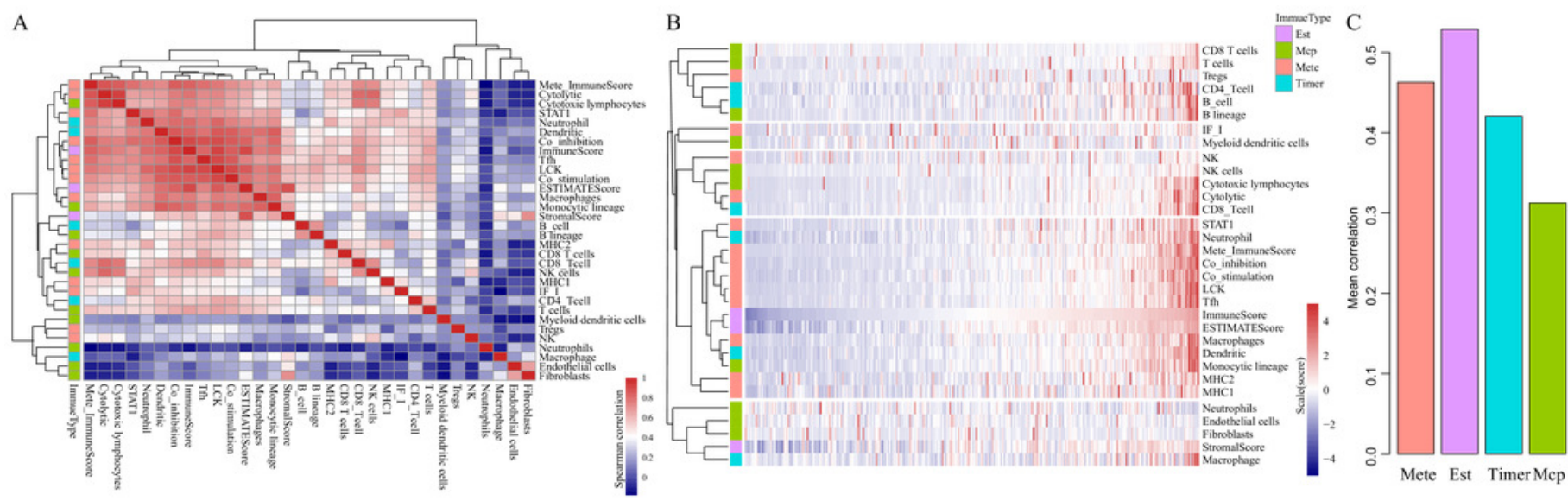
Figure 3

StromalScore (A), ImmuneScore (B) and ESTIMATEScore (C) distribution among CSCC patients with or without HPV infection.
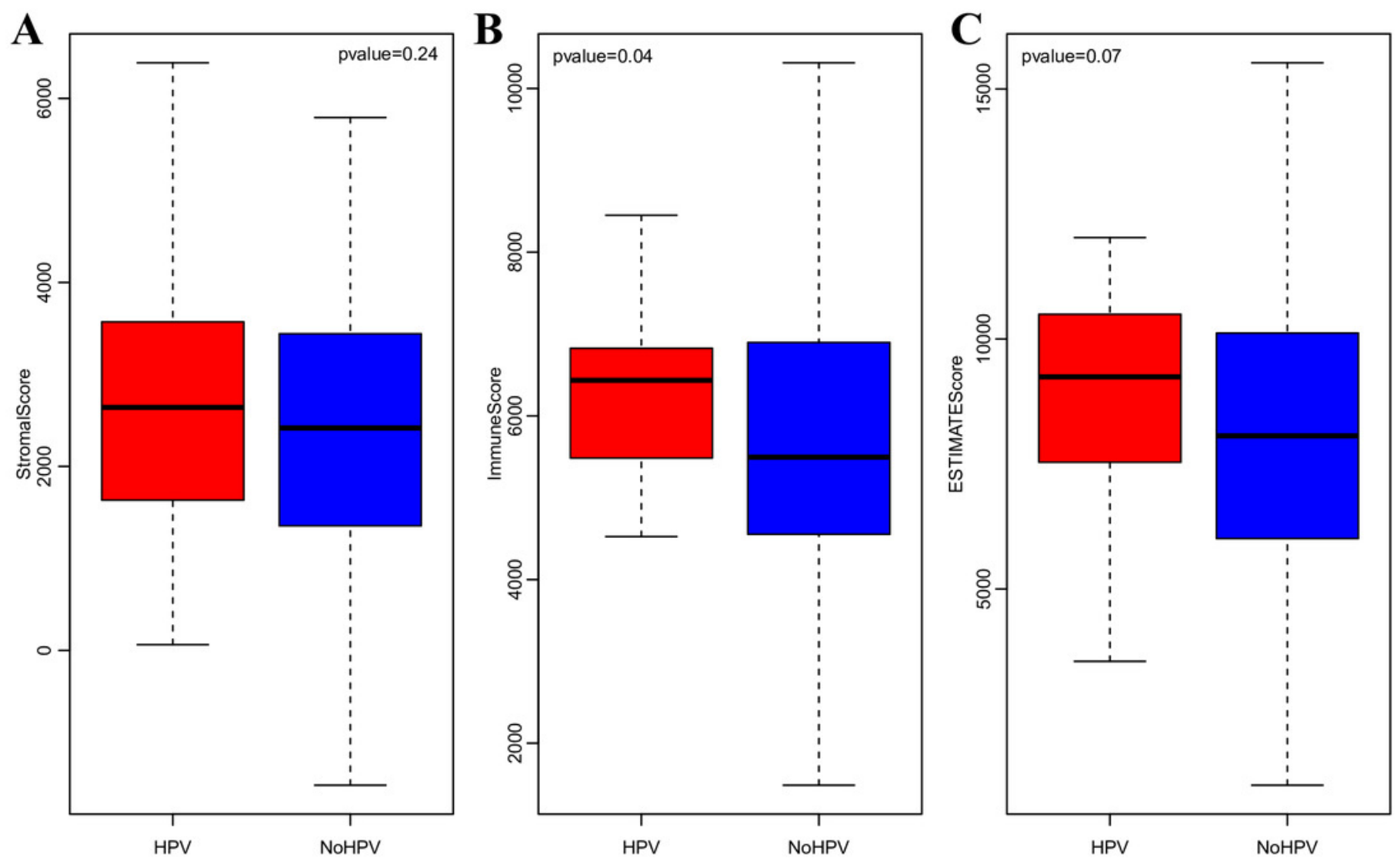
Figure 4

The relationships between levels of StromalScore (A), ImmuneScore (B) or ESTIMATEScore $(C)$ and prognosis for CSCC patients.

H:High immune score; L: Low immune score.
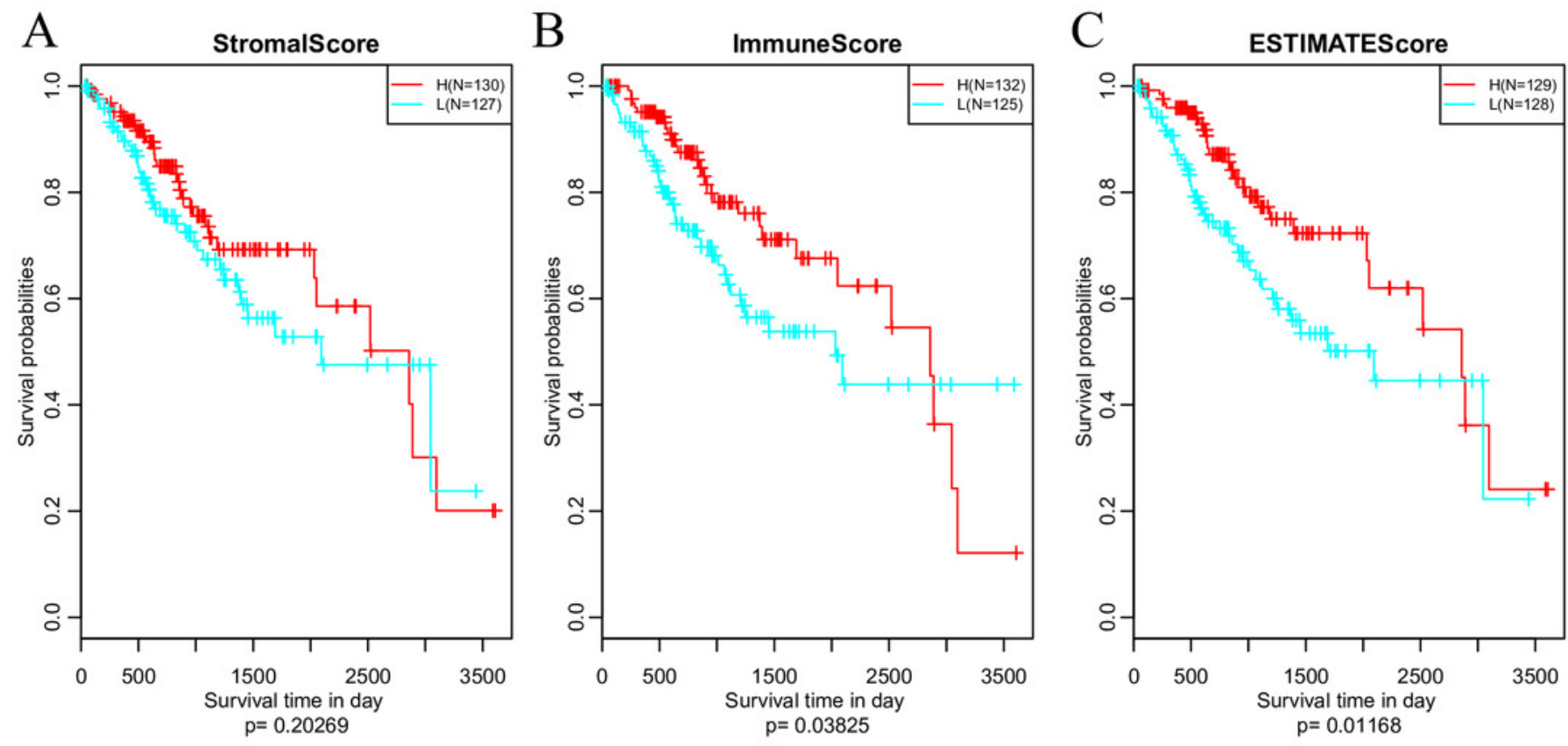


\section{Figure 5}

The correlations of immune-related scores based on ESTIMATE algorithm with gene mutations.

The StromalScore $(A, D, G, J)$, ImmuneScore $(B, E, H, K)$ and ESTIMATEScore $(C, F, I, L)$ were calculated respectively in HLA-A (A, B, C), HLA-B (D, E, F), HLA-C (G, H, I) and TP53 (J, K, L) mutation and non-mutation groups. Green represents the mutant group and red represents the wild type. Mut: Mutant; WT: Wild type. 

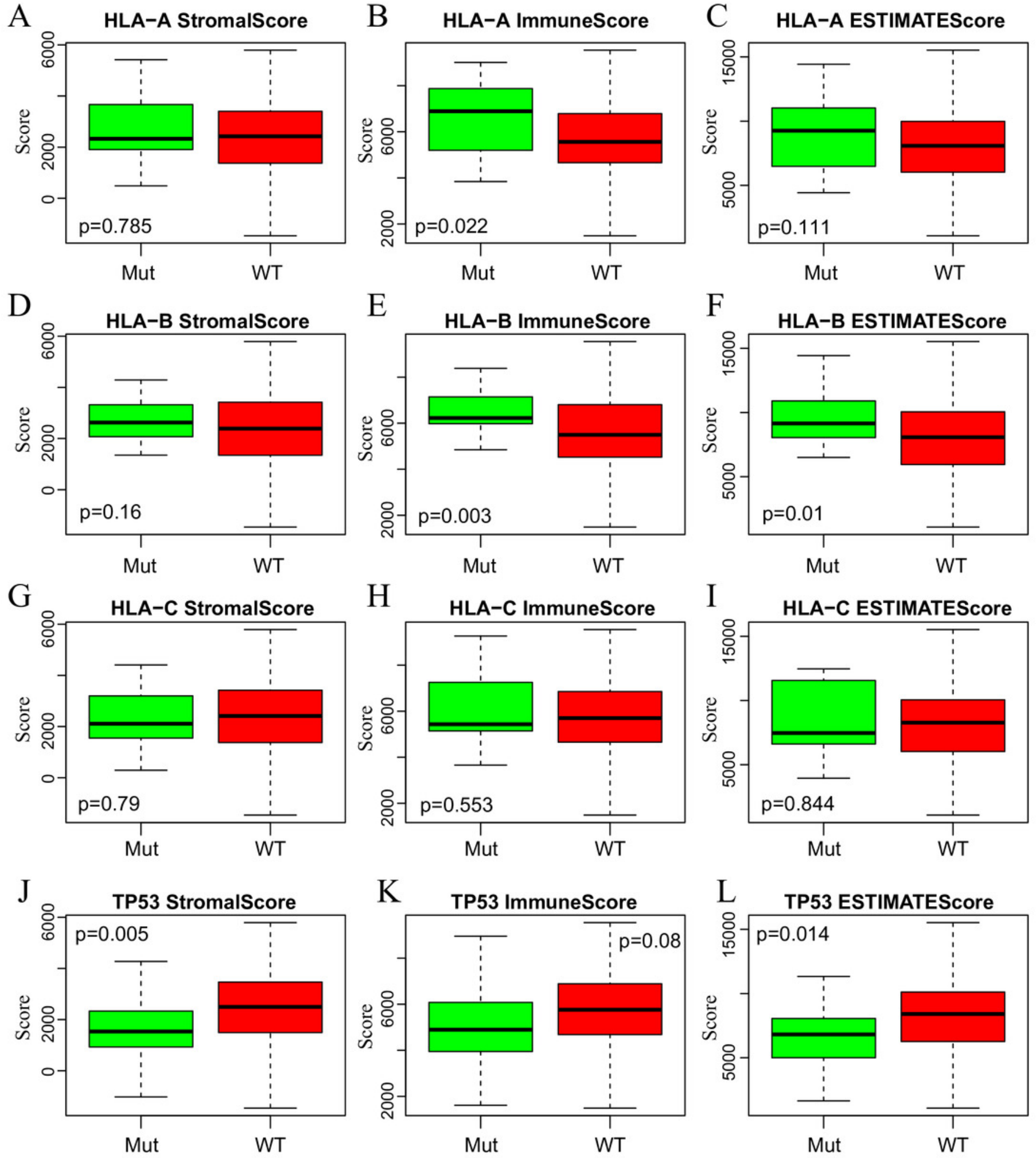
Figure 6

Immune scores-related gene modules mined through WGCNA

(A) Sample clustering analysis. (B, C) Analysis of network topology for various soft-

thresholding powers. (D) Gene dendrogram and module colors. (E) Correlation between each module and three immune-related scores.

A

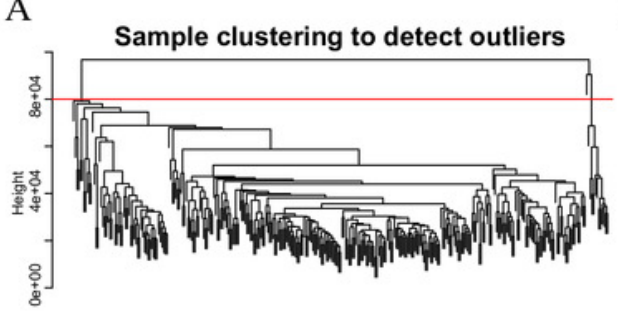

B

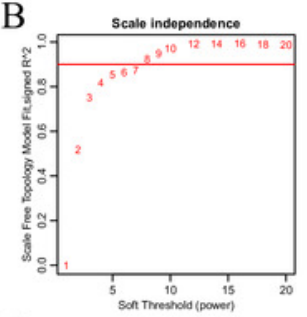

E

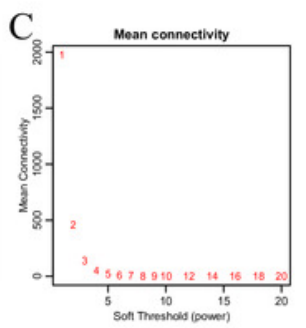

5 Solt Thesinous (comerer)
D

Cluster Dendrogram

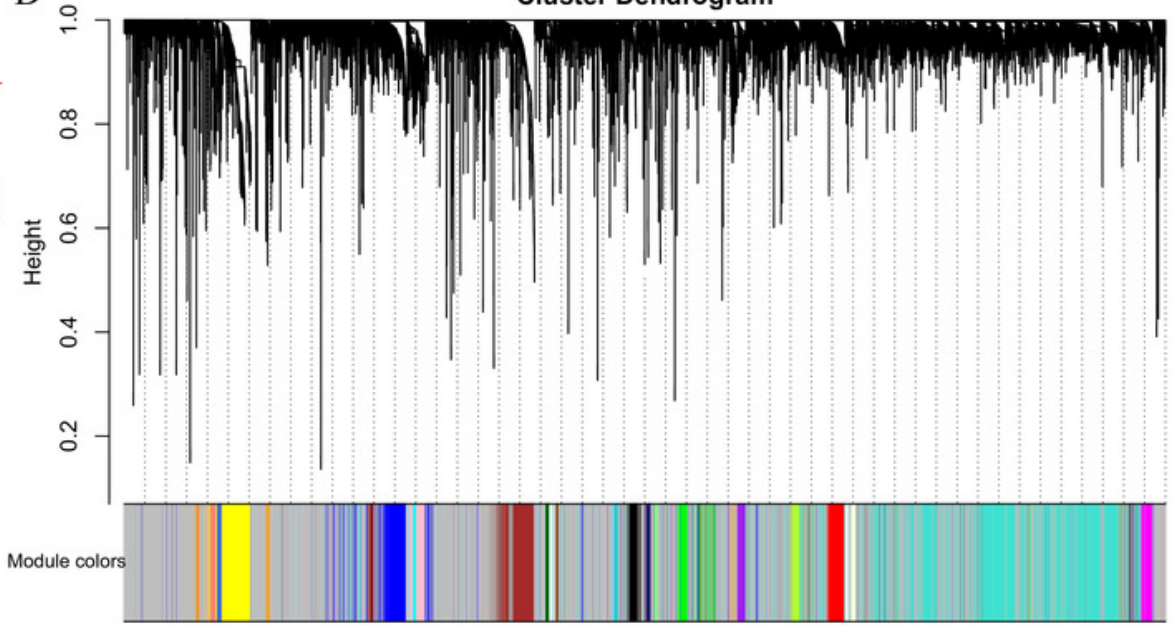

Module-trait relationships

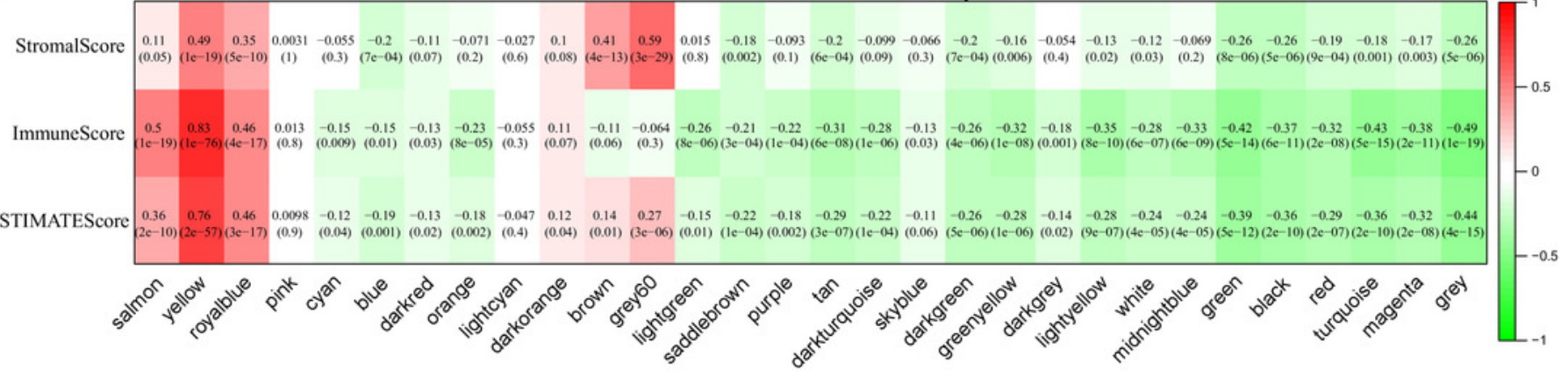




\section{Figure 7}

The KEGG pathway and GO enrichment analysis of the genes in yellow module.

(A) Top20 KEGG pathways enriched by the genes in yellow module. (B) Top20 GO BP terms enriched by the genes in yellow module. (C) Top20 GO CC terms enriched by the genes in yellow module. (D) Top20 GO MF terms enriched by the genes in yellow module. GO: Gene Ontology; BP: biological process; CC: cellular component; MF: molecular function 
A

C
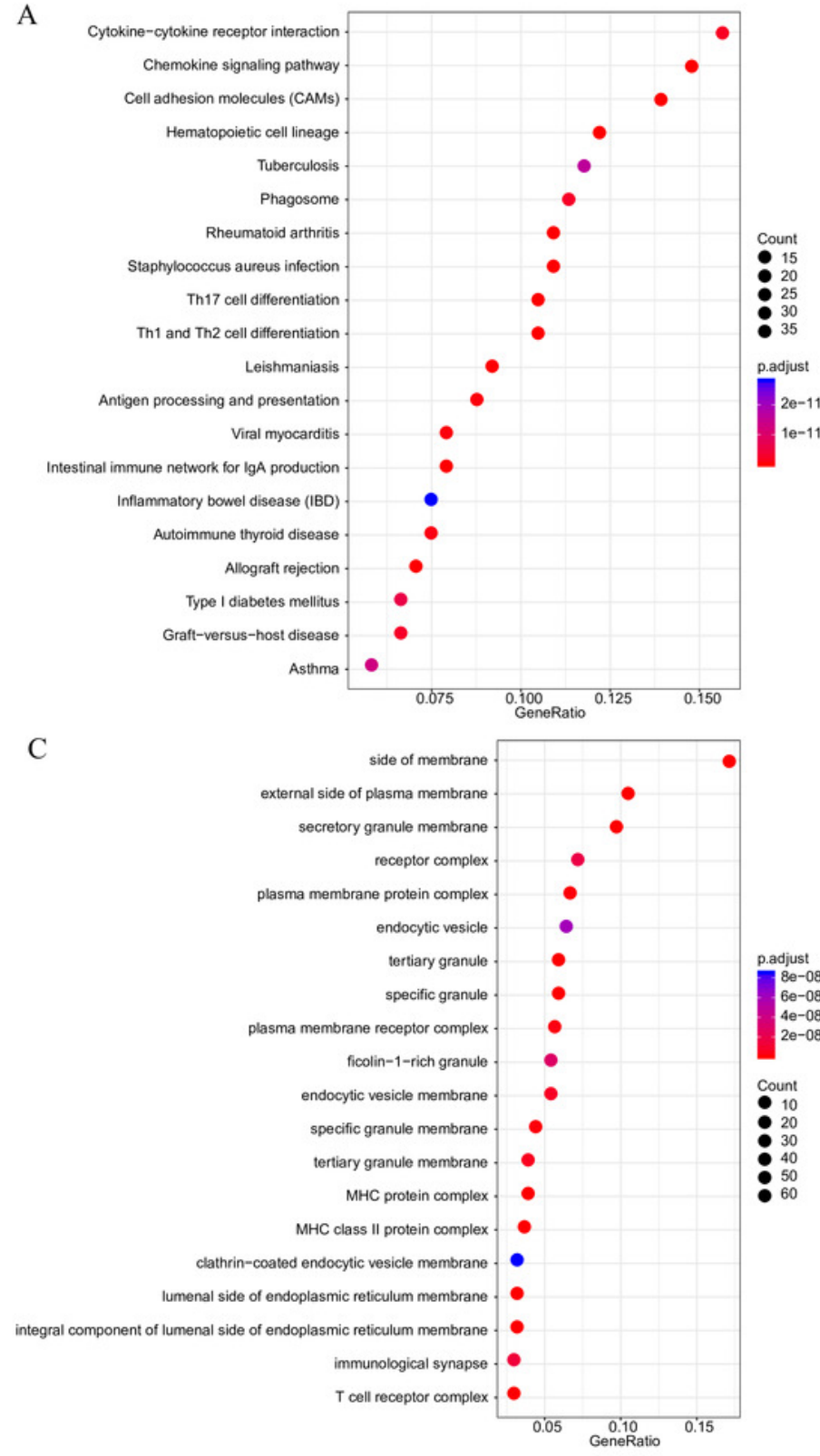

B
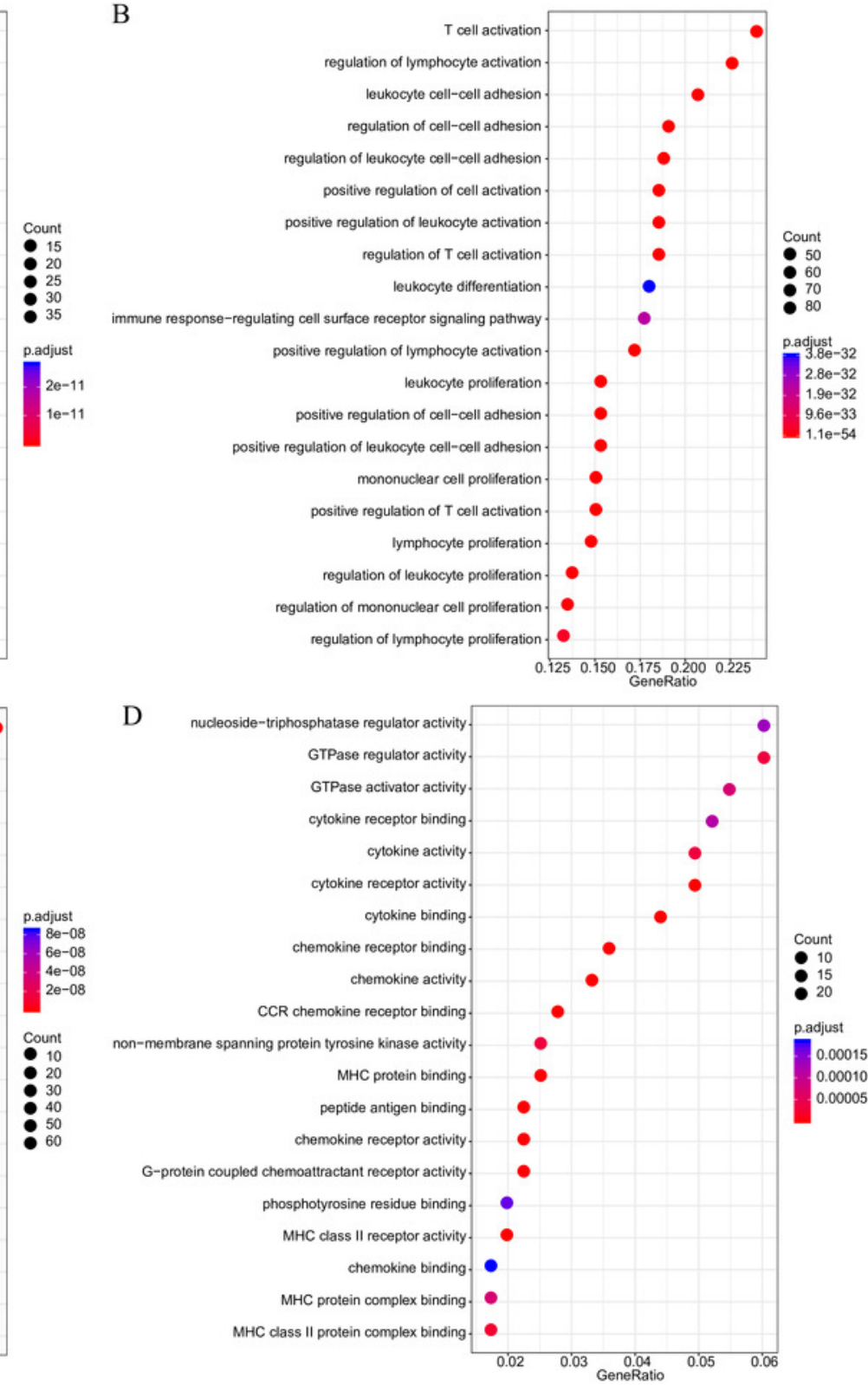

D

non-membrane spanning protein tyrosine kinase activity

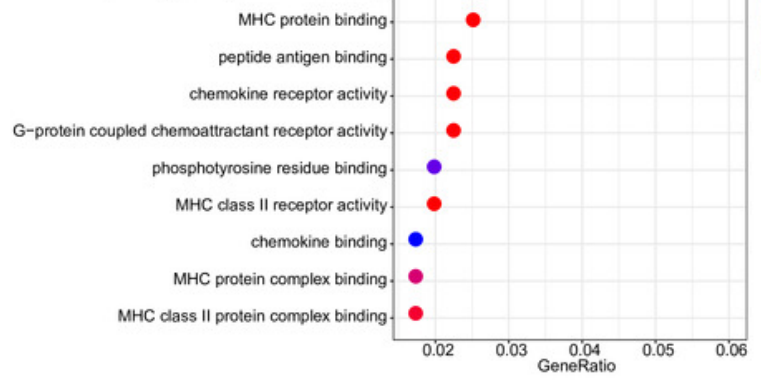

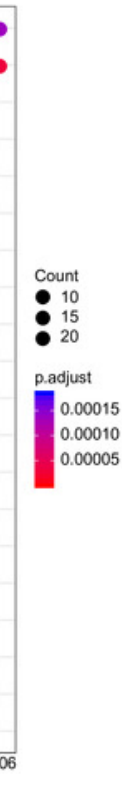




\section{Figure 8}

Construction of co-expression network of yellow module-related genes.

(A) Co-expression network of weights between genes in yellow module. (B) The degree distribution of nodes in yellow module. (C) The correlation of genes and module in the network.
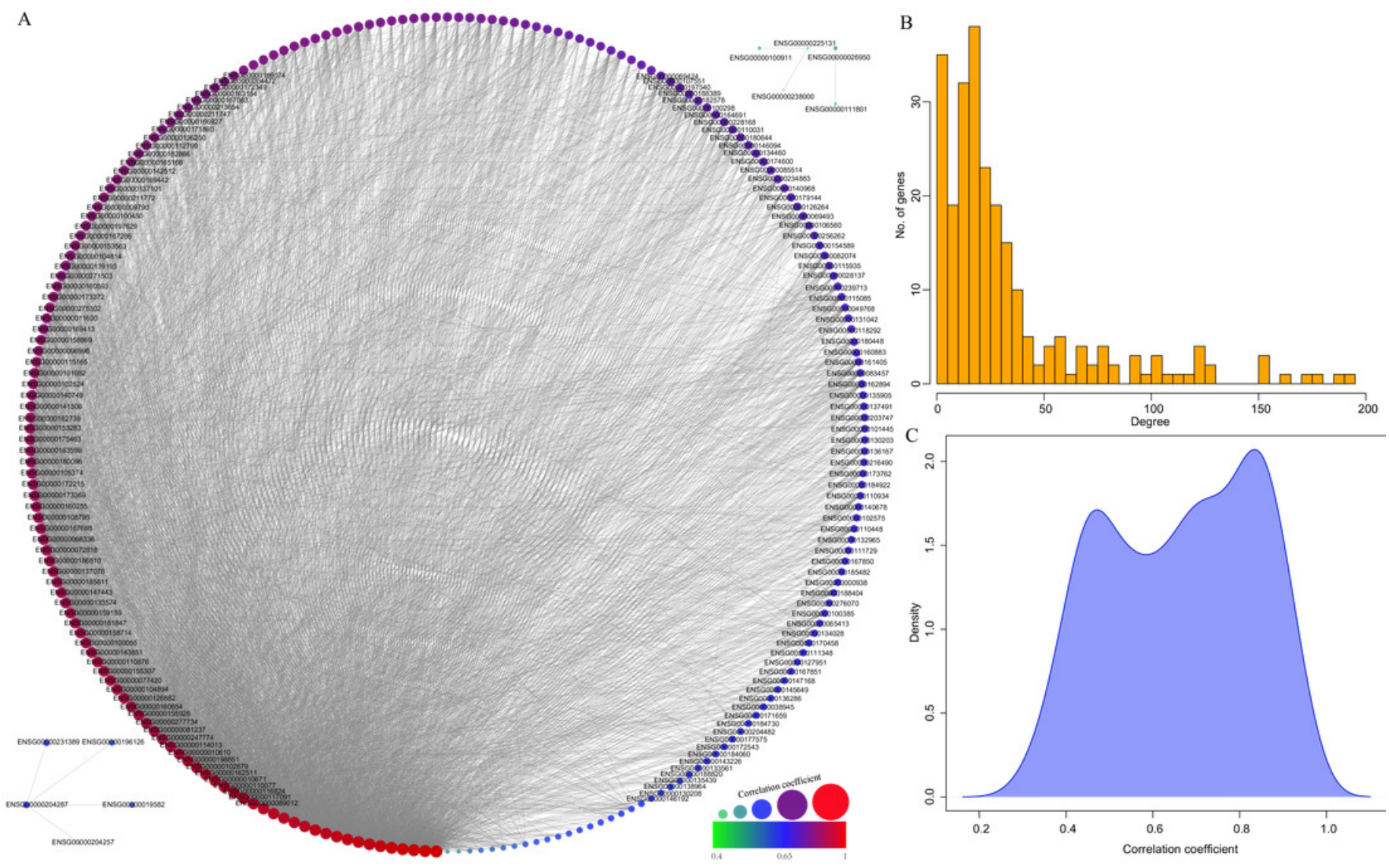
Figure 9

The KEGG pathway and GO enrichment analysis of 18 novel representative immune microenvironment-related genes for CSCC patients.

(A) Top20 KEGG pathways enriched by 18 novel representative immune microenvironmentrelated genes. (B) Top20 GO BP terms enriched by 18 novel representative immune microenvironment-related genes. (C) Top20 GO CC terms enriched by 18 novel representative immune microenvironment-related genes. (D) Top20 GO MF terms enriched by 18 novel representative immune microenvironment-related genes. GO: Gene Ontology; BP: biological process; CC: cellular component; MF: molecular function
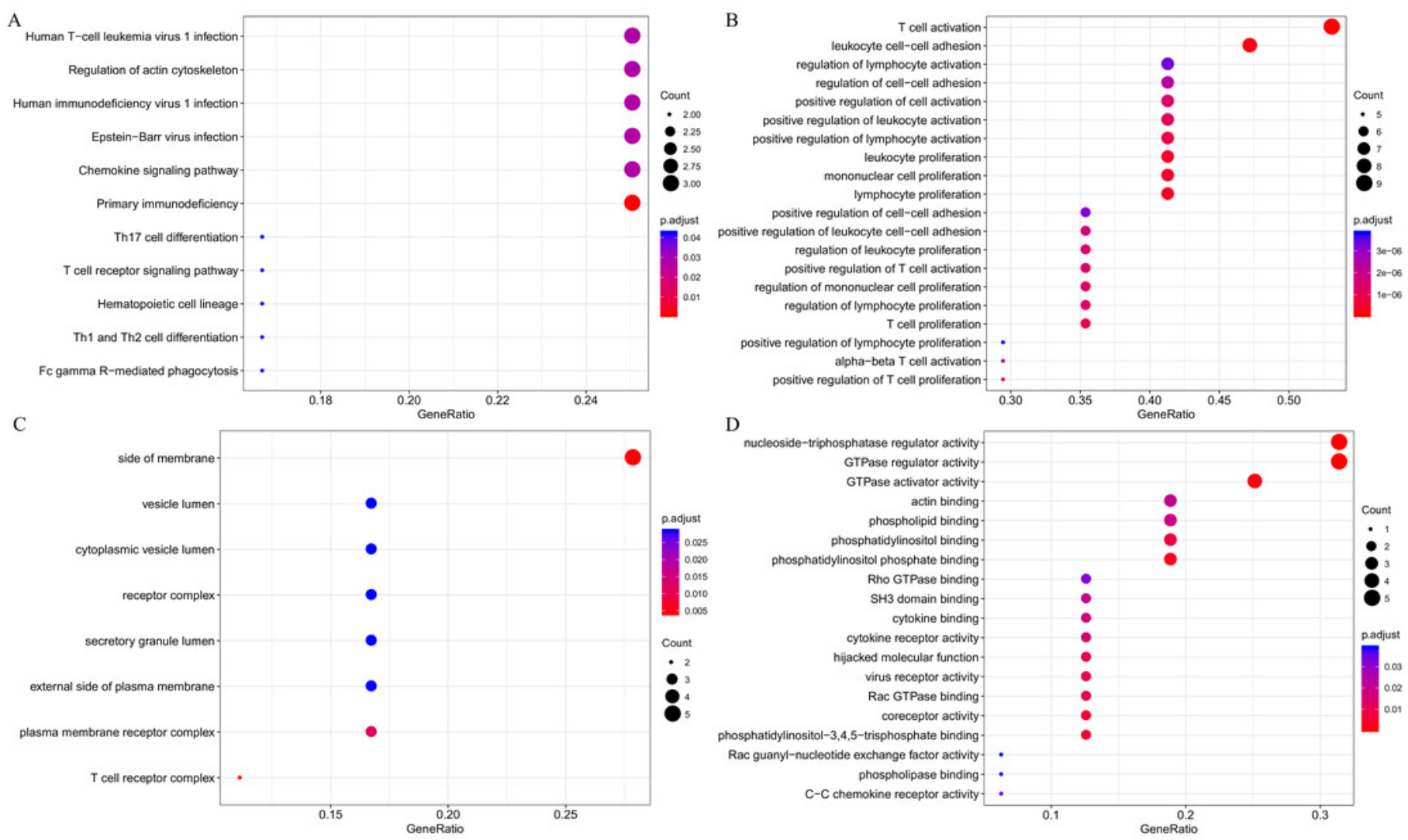


\section{Figure 10}

Microsoft Word - manuscript change-tracked.docx The association between 18 novel representative immune microenvironment-related genes for CSCC patients and immune checkpoints.

Apart from CD276 and VTCN1, the other 6 immune checkpoints were significantly related to 18 immune microenvironment-related genes.

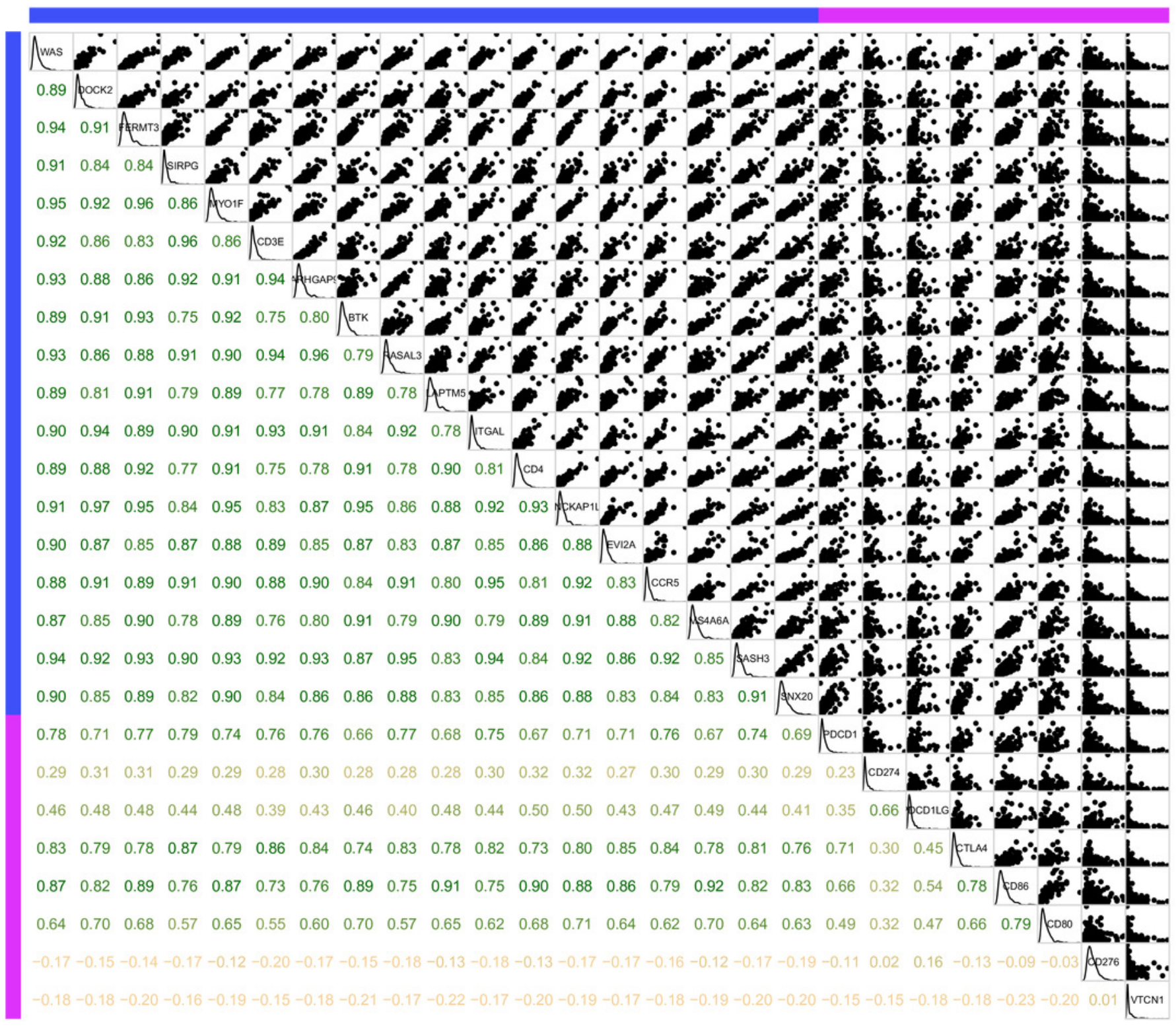


Figure 11

The relationship between 18 novel representative immune microenvironment-related genes and prognosis (A-P)

Apart from DOCK2 ( $P=0.12765)$, SIRPG $(P=0.0658), C D 4(P=0.29192), N C K A P 1 L(P=0.12765)$ and CCR5 ( $P=0.06736)$, high expression of other 13 genes were significantly associated with better overall survival.
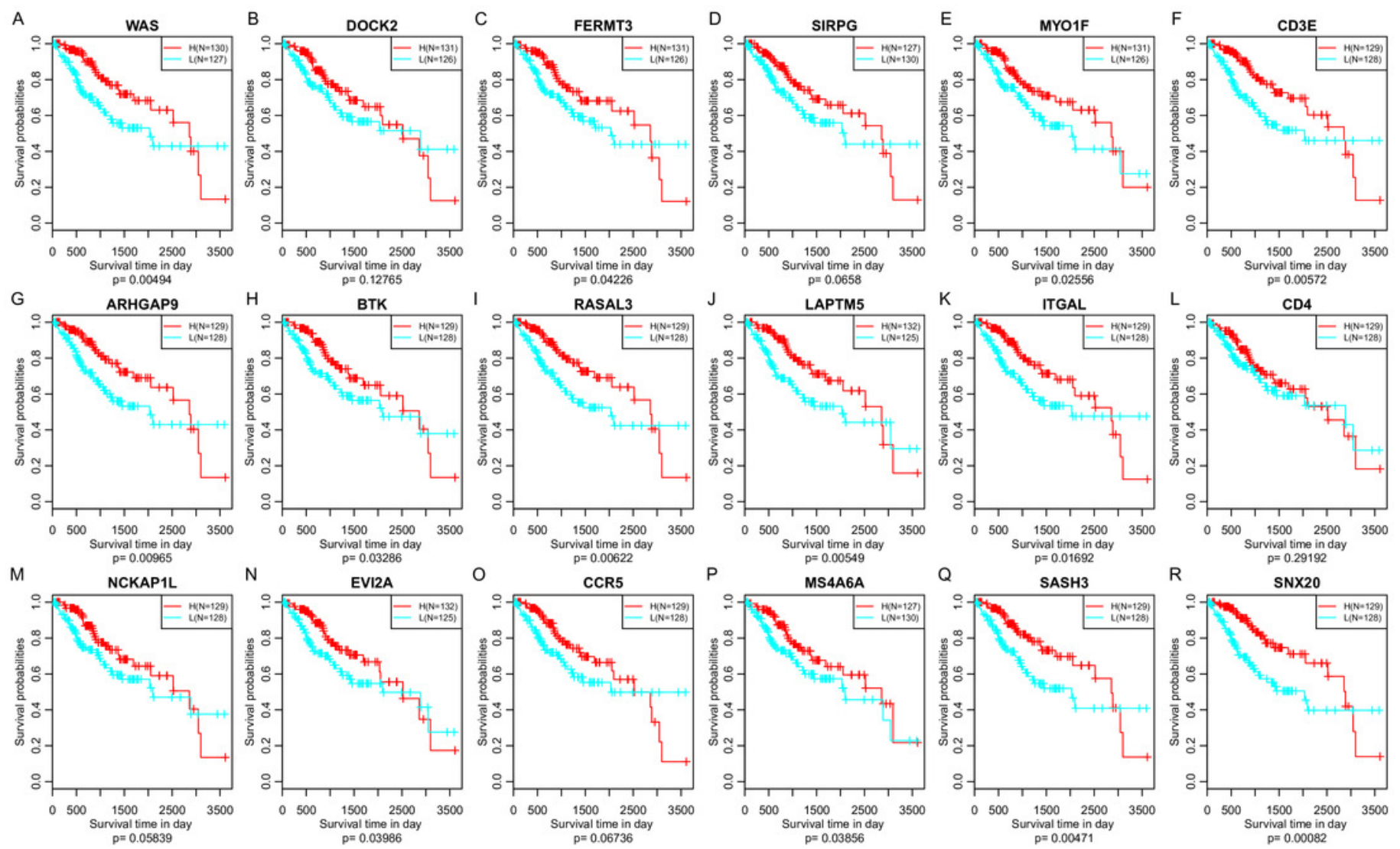
Figure 12

The correlations of 18 immune microenvironment-related genes with ImmuneScore for CSCC patients in independent dataset (A-P).

Apart from CCR5 ( $P=0.867, R=0.01)$, the other 17 genes were significantly associated with the ImmuneScore.
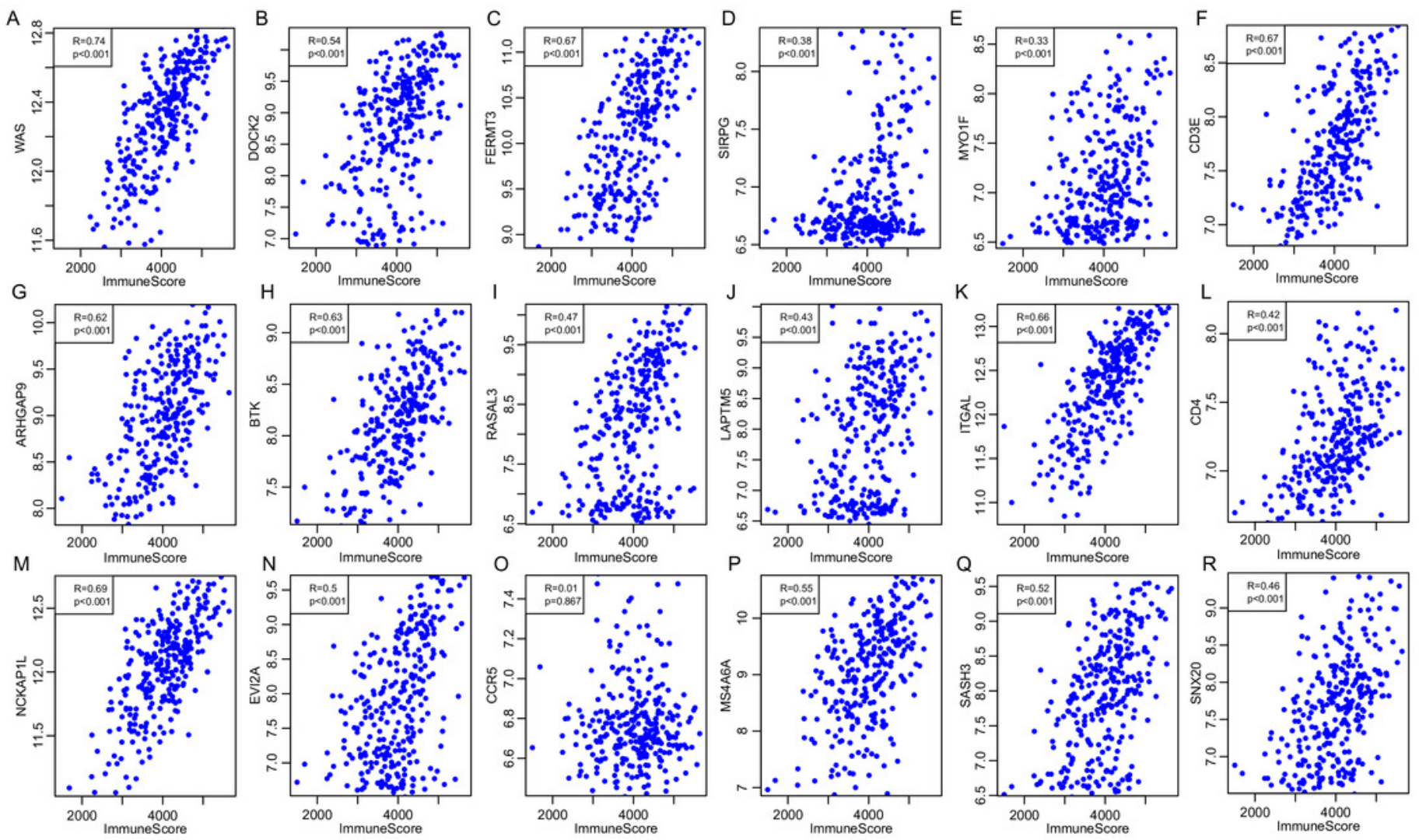


\section{Table $\mathbf{1}$ (on next page)}

Number of transcripts in each module 
1 Table 1 Number of transcripts in each module

\begin{tabular}{|c|c|}
\hline Modules & Genes \\
\hline Black & 232 \\
\hline Blue & 676 \\
\hline Brown & 469 \\
\hline Cyan & 82 \\
\hline Darkgreen & 53 \\
\hline Darkgrey & 44 \\
\hline Darkorange & 38 \\
\hline Darkred & 54 \\
\hline Darkturquoise & 47 \\
\hline Green & 276 \\
\hline Greenyellow & 110 \\
\hline Grey & 7417 \\
\hline Grey 60 & 67 \\
\hline Lightcyan & 68 \\
\hline Lightgreen & 66 \\
\hline Lightyellow & 65 \\
\hline Magenta & 181 \\
\hline Midnightblue & 78 \\
\hline Orange & 40 \\
\hline Pink & 232 \\
\hline Purple & 116 \\
\hline Red & 261 \\
\hline Royalblue & 64 \\
\hline Saddlebrown & 31 \\
\hline Salmon & 97 \\
\hline Skyblue & 33 \\
\hline Tan & 98 \\
\hline Turquoise & 2642 \\
\hline
\end{tabular}


White

Yellow

422

2 


\section{Table 2 (on next page)}

Genes with a correlation over 0.9 and a degree over 50 in the network 
1 Table 2 Genes with a correlation over 0.9 and a degree over 50 in the network

\begin{tabular}{|c|c|c|c|c|}
\hline ENSG & Symbol & corr.R & Degree & MeteGene \\
\hline ENSG00000015285 & WAS & 0.964019 & 188 & \\
\hline ENSG00000110324 & IL10RA & 0.944217 & 154 & $\mathrm{LCK}$ \\
\hline ENSG00000134516 & DOCK2 & 0.932541 & 113 & \\
\hline ENSG00000149781 & FERMT3 & 0.957826 & 171 & \\
\hline ENSG00000043462 & $\mathrm{LCP} 2$ & 0.941048 & 102 & CLK \\
\hline ENSG00000185862 & EVI2B & 0.94047 & 153 & LCK \\
\hline ENSG00000117091 & CD48 & 0.918649 & 107 & $\mathrm{LCK}$ \\
\hline ENSG00000089012 & SIRPG & 0.918974 & 119 & \\
\hline ENSG00000135077 & HAVCR2 & 0.932432 & 95 & Co_inhibition \\
\hline ENSG00000116824 & CD2 & 0.915954 & 124 & LCK \\
\hline ENSG00000142347 & MYO1F & 0.962694 & 193 & \\
\hline ENSG00000198851 & CD3E & 0.90917 & 130 & \\
\hline ENSG00000123329 & ARHGAP9 & 0.925285 & 126 & \\
\hline ENSG00000010671 & BTK & 0.913087 & 85 & \\
\hline ENSG00000105122 & RASAL3 & 0.92036 & 124 & \\
\hline ENSG00000162511 & LAPTM5 & 0.912211 & 72 & \\
\hline ENSG00000005844 & ITGL & 0.92691 & 125 & \\
\hline ENSG00000010610 & CD48 & 0.908066 & 57 & \\
\hline ENSG00000123338 & NCKAP1L & 0.953232 & 162 & \\
\hline ENSG00000102879 & CORO1A & 0.909449 & 94 & LCK \\
\hline ENSG00000126860 & EVI2A & 0.923238 & 70 & \\
\hline ENSG00000143119 & CD53 & 0.957049 & 178 & $\mathrm{LCK}$ \\
\hline ENSG00000160791 & CCR5 & 0.926682 & 104 & \\
\hline ENSG00000110077 & MS4A6A & 0.915621 & 57 & \\
\hline ENSG00000122122 & SASH3 & 0.949558 & 153 & \\
\hline ENSG00000167208 & SNX20 & 0.942276 & 123 & $\square$ \\
\hline
\end{tabular}

